\title{
Relative abundance pattern along the profile of high redshift Damped Lyman- $\alpha$ systems ${ }^{\star}$
}

\author{
E. Rodríguez ${ }^{1}$, P. Petitjean ${ }^{1,2}$, B. Aracil ${ }^{1,3}$, C. Ledoux $^{4}$, and R. Srianand ${ }^{5}$ \\ ${ }^{1}$ Institut d'Astrophysique de Paris, UMR 7095, 98 bis boulevard Arago, 75014 Paris, France \\ e-mail: rodrig@iap.fr \\ 2 Observatoire de Paris, LERMA, UMR 8112, 61 avenue de l'Observatoire, 75014 Paris, France \\ 3 Department of Astronomy, University of Massachusetts, 710 North Pleasant Street, Amherst, MA 01003-9305, USA \\ ${ }^{4}$ European Southern Observatory, Alonso de Cordova 3107, Casilla 19001, Vitacura, Santiago, Chile \\ 5 IUCAA, Post Bag 4, Ganesh Khind, Pune 411 007, India
}

Received 8 December 2004 / Accepted 23 August 2005

\section{ABSTRACT}

We investigated abundance ratios along the profiles of six high-redshift Damped Lyman- $\alpha$ systems, three of them associated with $\mathrm{H}_{2}$ absorption, and derived optical depths in each velocity pixel. The variations of the pixel abundance ratios were found to be remarkably small and usually smaller than a factor of two within a profile. This result holds even when considering independent sub-clumps in the same system. The depletion factor is significantly enhanced only in those components where $\mathrm{H}_{2}$ is detected. There is a strong correlation between [Fe/S] and $[\mathrm{Si} / \mathrm{S}]$ abundances ratios, showing that the abundance ratio patterns are definitely related to the presence of dust. The depletion pattern is usually close to the one seen in the warm halo gas of our Galaxy.

Key words. cosmology: observations - quasars: individual: Q 0528-250, Q 0013+004, Q 1037-270, Q 1157+014, Q 0405-443

\section{Introduction}

Damped Lyman- $\alpha$ systems (hereafter DLAs) observed in QSO spectra are characterized by strong H I $\lambda 1215$ absorption lines with broad damping wings. Although the definition has been restricted for historical reasons to absorptions with $\log N(\mathrm{H} \mathrm{I})>20.3$ (Wolfe et al. 1986), damping wings are easily detected in present-day, high quality data for much lower column densities (down to $\log N(\mathrm{H} \mathrm{I}) \sim 18.5$ ). A more appropriate definition should be related to the physical state of the gas. If we impose the condition that the gas must be neutral, then the definition should be limited to systems with $\log N(\mathrm{H} \mathrm{I})>19.5$ (e.g. Viegas 1995).

Since their discovery twenty years ago (Wolfe et al. 1986), DLAs clearly have something to do with galaxy formation. What kind of galaxy DLAs are associated to is, however, still a matter of debate. Some authors identify these systems with large rotating discs (Prochaska \& Wolfe 1997; Hou et al. 2001), while others think that DLAs arise mostly either in dwarf galaxies (Centuríon et al. 2000) or galactic building blobs

* Based on observations carried out at the European Southern Observatory (ESO), under progs. ID 65.O-0063, 66.A-0624, 67.A-0078, 68.A-0106 and 68.A-0600, with the UVES echelle spectrograph installed at the Very Large Telescope (VLT) unit KUEYEN on Mount Paranal, Chile.
(Haehnelt et al. 1998; Ledoux et al. 1998). The answer is probably not unique. In any case, DLAs represent the major reservoir of neutral hydrogen at any redshift (Storrie-Lombardi \& Wolfe 2000), and they probe the chemical enrichment and evolution of the neutral Universe (see Pettini et al. 1994; Lu et al. 1996; Prochaska et al. 1999; Ledoux et al. 2002a, and references therein). Since abundances can be measured very accurately in DLAs, we can both discuss the connection between observed abundance ratios and dust content and to trace the nucleosynthesis history of the dense gas in the universe.

In this context, it is helpful to compare these results with measurements in the ISM of our Galaxy. Refractory elements that condense easily into dust grains - namely, $\mathrm{Cr}, \mathrm{Fe}, \mathrm{Ni}$ - are strongly depleted (up to a factor hundred) in the ISM, while non-refractory elements remain in its gaseous phase $-\mathrm{S}, \mathrm{Zn}$, and partially $\mathrm{Si}-$. The amount of depletion depends on the physical condition of the gas. Thus, different depletion patterns are observed depending on whether the gas is cold or warm and/or whether the gas is located in the disc or the halo of the Galaxy (Savage \& Sembach 1996). The LMC and SMC also exhibit different gas-phase abundance ratios (Welty et al. 1999).

However, a particular nucleosynthesis history can give rise to peculiar metallicity patterns and mimic the presence of dust. Tinsley (1979) suggested that type Ia supernova are the major 
producers of Fe. An enhancement in $[\alpha / \mathrm{Fe}]$ ratios ( $\alpha$-elements are mostly $\mathrm{O}, \mathrm{S}, \mathrm{Si}$ ) could reflect an IMF skewed to high masses and therefore a predominant role of type II supernova. For very low metallicity stars $([\mathrm{Fe} / \mathrm{H}]<-3)$ in the Galaxy, large variations in several abundance ratios have been reported (McWilliam 1997), which suggests that peculiar nucleosynthesis processes and inhomogeneous chemical enrichment are probably taking place.

As mentioned above, DLAs trace the chemical evolution of galaxies at early epochs in the universe. Many detailed studies have been performed so far, revealing that their metallicities range between $1 / 300 Z_{\odot}$ and solar values. The abundance pattern is fairly uniform and compatible with low dust content (see Pettini et al. 1994; Lu et al. 1996; Prochaska et al. 1999; Ledoux et al. 2002a). This uniformity in the relative abundance patterns observed from one DLA to the other has been emphasized by Prochaska \& Wolfe (2002) and suggests that protogalaxies have common enrichment histories.

Few studies have adressed the question of the homogeneity inside each particular system. Prochaska \& Wolfe (1996) first studied chemical abundance variations in a single DLA, and showed that the chemical abundances were uniform to within statistical uncertainties. Lopez et al. (2002) confirmed this finding from analysis of another DLA using Voigt profile decomposition. Petitjean et al. (2002) and Ledoux et al. (2002b) showed that the depletion patterns in subcomponents were very similar along DLA profiles except in the components where molecular hydrogen is detected and where depletion is larger. More recently, Prochaska (2003), performed a study of 13 systems concluding that the majority of DLAs have very uniform relative abundances. This contrasts in particular with the dispersion in nucleosynthetic enrichment of the Milky Way as traced by stellar abundances. Here, we use the best data from our survey of DLAs (Ledoux et al. 2003) to investigate this issue further using an inversion method to derive the velocity profiles in different abundance ratios. In particular, we investigate the consequence of the presence of molecular hydrogen in some of the DLAs. The paper is structured as follows: we describe the data in Sect. 2; in Sect. 3 we briefly introduce the method used for the analysis; and results are presented and discussed in Sects. 4 and 5.

\section{Observations and sample}

As emphasized by Prochaska (2003), a high S/N ratio is needed to investigate variations along the profile. In addition, we want to investigate the difference between systems where molecular hydrogen is and where is not detected because it has been shown (e.g. Petitjean et al. 2002) that depletion is larger in components where $\mathrm{H}_{2}$ is detected. We therefore restricted the sample to the six highest $\mathrm{S} / \mathrm{N}$ ratio spectra from the list of 33 quasars (24 DLAs and 9 sub-DLAs) observed during the VLT survey for molecular hydrogen in DLAs (Ledoux et al. 2003). The QSOs were observed with the Ultraviolet and Visible Echelle Spectrograph (D'Odorico et al. 2000), mounted on the $8.2 \mathrm{~m}$ Kueyen telescope operated at Cerro Paranal, Chile, during the observation periods P65 to P68. The actual spectral resolution lies in the range $42500<R<53000$.
For all the spectra, the $\mathrm{S} / \mathrm{N}$ ratio is larger than 50 per pixel (see Ledoux et al. 2003, for details). For Q 0528-250, we also retrieved complementary data from the ESO archive, thereby obtaining a very high-quality spectrum with $S N R \sim$ 100. Molecular hydrogen was detected in three of these systems. This is the case for Q 0013-004 (Petitjean et al. 2002), Q 0528-250 (Srianand \& Petitjean 1998), and Q 0405-443 -system at $z_{\mathrm{abs}}=2.595-($ Ledoux et al. 2003). The six systems span a wide range of $\mathrm{H}$ I column densities from $\log N(\mathrm{H} \mathrm{I})=19.7$ up to 21.80 .

In all systems we chose to analyze absorption features that are well-defined and do not suffer from major blending.

\subsection{Q 0013-004}

The presence of $\mathrm{H}_{2}$ at $z_{\mathrm{abs}}=1.973$ was first reported by Ge \& Bechtold (1997). Spread over more than $1000 \mathrm{~km} \mathrm{~s}^{-1}$ (see Fig. 7), this system is the DLA absorber with the highest molecular fraction known so far $\left(-2.7<f\left(\mathrm{H}_{2}\right)<-0.64\right.$, Petitjean et al. 2002) for $\log N(\mathrm{H} \mathrm{I})=20.83$. Molecular hydrogen has been detected in four different components spread all over the system, at relative velocities, $\sim 615, \sim-480, \sim 0$ and $\sim+80 \mathrm{~km} \mathrm{~s}^{-1}$. Petitjean et al. (2002) also report four additional strong metal components that probably make a nonnegligible contribution to the total $\mathrm{H}$ I column density. All components have similar abundance ratios and depletion factors independent of the presence or absence of $\mathrm{H}_{2}$. Only for the special molecular component at $z_{\mathrm{abs}}=1.97296$ does the dust depletion turn to be important. The depletion of $\mathrm{Fe}$ is comparable to that observed in the cold interstellar medium of the Galactic disc. We summarize the overall abundances of the principal elements present in the system in Table 4 . We also confirm the strong depletion pattern in the $\sim 480 \mathrm{~km} \mathrm{~s}^{-1}$ component.

\section{2. $Q 0405-443$}

Lopez et al. (2001) first discovered three DLA systems along the line of sight to this quasar at redshifts $z_{\mathrm{abs}}=2.550,2.595$ and 2.621. Later, Ledoux et al. (2003) confirmed the damped nature of these three absorptions. We adopted their H I column density values. From the three systems observed in this line of sight, we included the systems at $z_{\mathrm{abs}}=2.549, \log N(\mathrm{H} \mathrm{I})=21.0$ and $z_{\text {abs }}=2.595, \log N(\mathrm{H} \mathrm{I})=20.9$ in our sample. In the third system, metallicity is much lower, $[\mathrm{Fe} / \mathrm{H}]=-2.15$, which renders the analysis much more uncertain. Ledoux et al. (2003) detect the presence of $\mathrm{H}_{2}$ at $z_{\mathrm{abs}}=2.595$ from $J=0,1,2,3$ rotational transitions. They measure $\log N\left(\mathrm{H}_{2}\right)=18.16$, one of the largest $\mathrm{H}_{2}$ column densities ever seen in DLAs, although the molecular fraction in the corresponding cloud is not all that large $\left(\log f\left(\mathrm{H}_{2}\right)=-2.44\right)$ due to the high column density of neutral hydrogen.

\subsection{PKS 0528-250}

We collected complementary data from the ESO archive available on this QSO and added them together with our own data. 


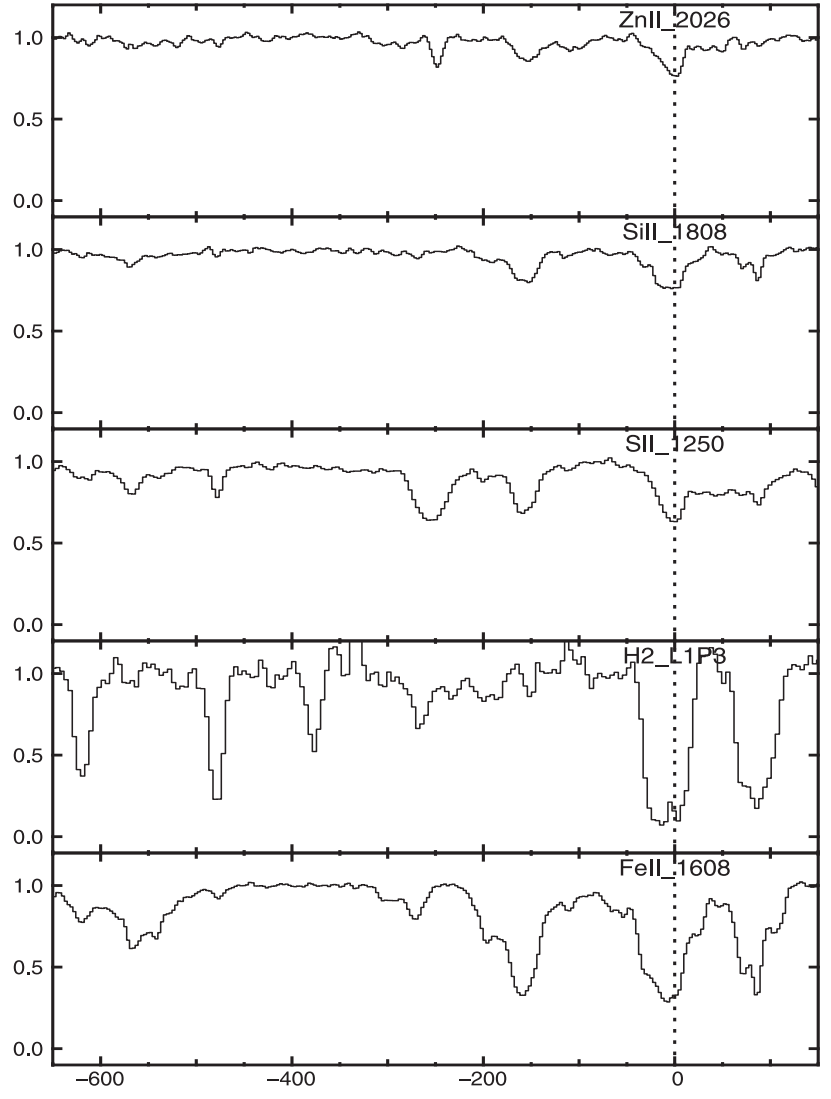

Fig. 1. Absorption spectra plotted on a velocity scale for a few transitions - including H2L1P3 - observed in the $z_{\text {abs }}=1.973$ DLA system toward Q 0013-004. Molecular transitions have been detected in four components located at $v \sim-615 \mathrm{~km} \mathrm{~s}^{-1}, v \sim-480 \mathrm{~km} \mathrm{~s}^{-1}$, $v \sim 0 \mathrm{~km} \mathrm{~s}^{-1}$, and $\sim 85 \mathrm{~km} \mathrm{~s}^{-1}$ (Petitjean et al. 2002).

The result is a very high $S N R$ spectrum that extends over the wavelength range 3000-10000 , apart from a few gaps. The absorption redshift is slightly higher than that of the emitting source, suggesting that the absorbing system could be associated with the quasar. A useful consequence of this is that a large number of metallic transitions are redshifted outside the Lyman- $\alpha$ forest and therefore can be used for our analysis. This system has been known for many years to be the only system at high redshift where molecules were detected (Levshakov \& Varshalovich 1985; Songaila \& Cowie 1996; Srianand \& Petitjean 1998). New data have been obtained with VLT by Ledoux et al. (2003). These authors derive $\log N\left(\mathrm{H}_{2}\right)=17.93$ and 18.0 in two components at $z_{\mathrm{abs}}=2.81100$ and 2.81112, respectively. Given the large neutral hydrogen column density $(\log N(\mathrm{H} \mathrm{I})=21.35)$, the molecular fraction is only $f\left(\mathrm{H}_{2}\right)=9 \times$ $10^{-4}$. The excitation temperature for the $J=1$ rotational level is between 150 and $200 \mathrm{~K}$, and the density is probably quite large (Srianand \& Petitjean 1998). As mentioned by Lu et al. (1996), the metal absorption lines are unusually wide and complex, spreading over about $400 \mathrm{~km} \mathrm{~s}^{-1}$. They appear to be structured in two main sub-clumps above and below $+140 \mathrm{~km} \mathrm{~s}^{-1}$ (see Fig. 4).

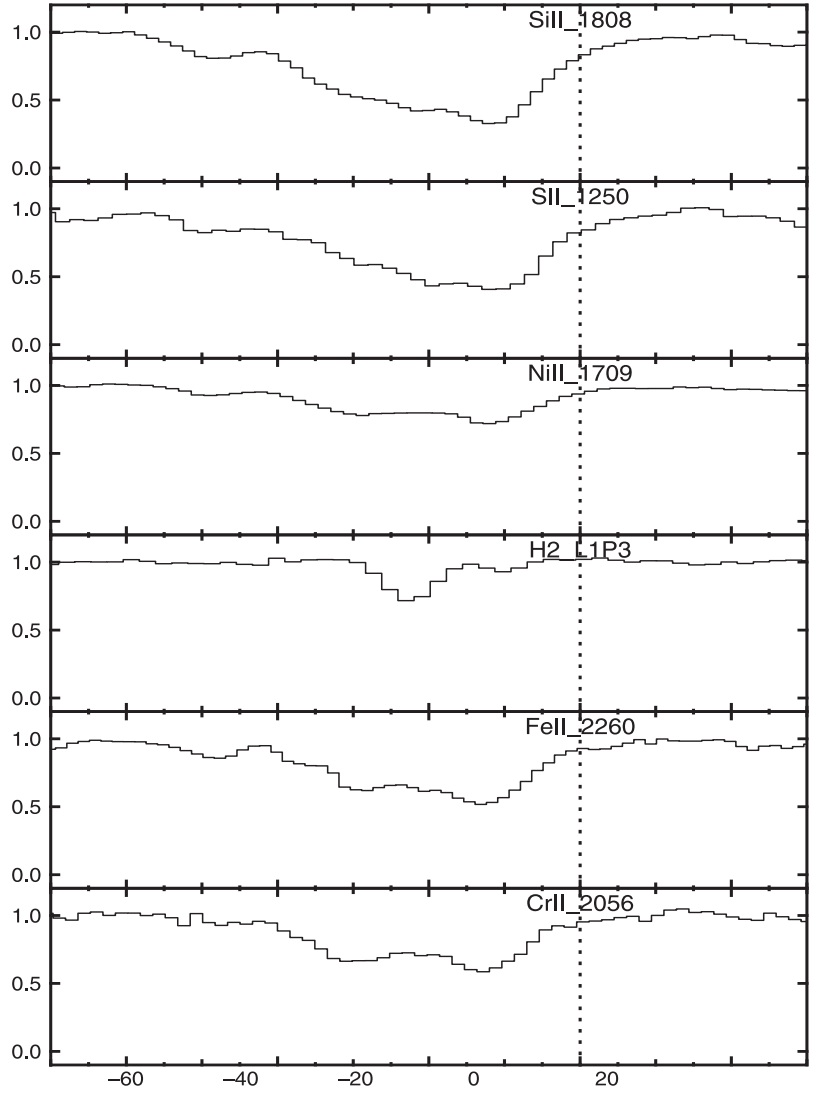

Fig. 2. Absorption spectra plotted on a velocity scale for a few transitions - including H2L1P3 transition - observed in the $z_{\mathrm{abs}}=2.595$ DLA system toward Q 0405-443. Numerous molecular transitions have been detected in two components located at $v \sim-24 \mathrm{~km} \mathrm{~s}^{-1}$ and $\sim-11 \mathrm{~km} \mathrm{~s}^{-1}$ (Ledoux et al. 2003).

\subsection{Q 1037-270}

The continuum of the QSO is difficult to constrain due to the presence of a complex system of BAL troughs (see Srianand $\&$ Petitjean 2001). A first limit on the neutral hydrogen column density was derived by Lespine \& Petitjean (1997) from the absence of damped wings in a low-resolution spectrum. We adopted $\log N(\mathrm{H} \mathrm{I})=19.7$ from Srianand \& Petitjean (2001). This is the lowest column density in our sample. However, this system has the highest metallicity known for DLAs at such a redshift $\left(z_{\mathrm{abs}}=2.139\right),[\mathrm{Zn} / \mathrm{H}]=-0.26$. As a consequence of this high metallicity, many low-ionization lines were detected, including C I, though no molecules were detected. Some of the corresponding species are shown in Fig. 5. The depletion pattern derived by Srianand \& Petitjean (2001) is compatible with very low dust content, if any at all. The gas seems to be warm and halo-like.

\section{5. $Q 1157+014$}

This system has the highest $\mathrm{H}$ I column density in our sample $(\log N(\mathrm{H} \mathrm{I})=21.8)$ and is close to the emission redshift of the quasar. Absorption in $21 \mathrm{~cm}$ has been detected by Briggs et al. (1984) and the spin temperature is constrained by Kanekar \& Chengalur (2003) to be $865 \pm 190 \mathrm{~K}$. Neither $\mathrm{H}_{2}$ nor C I 


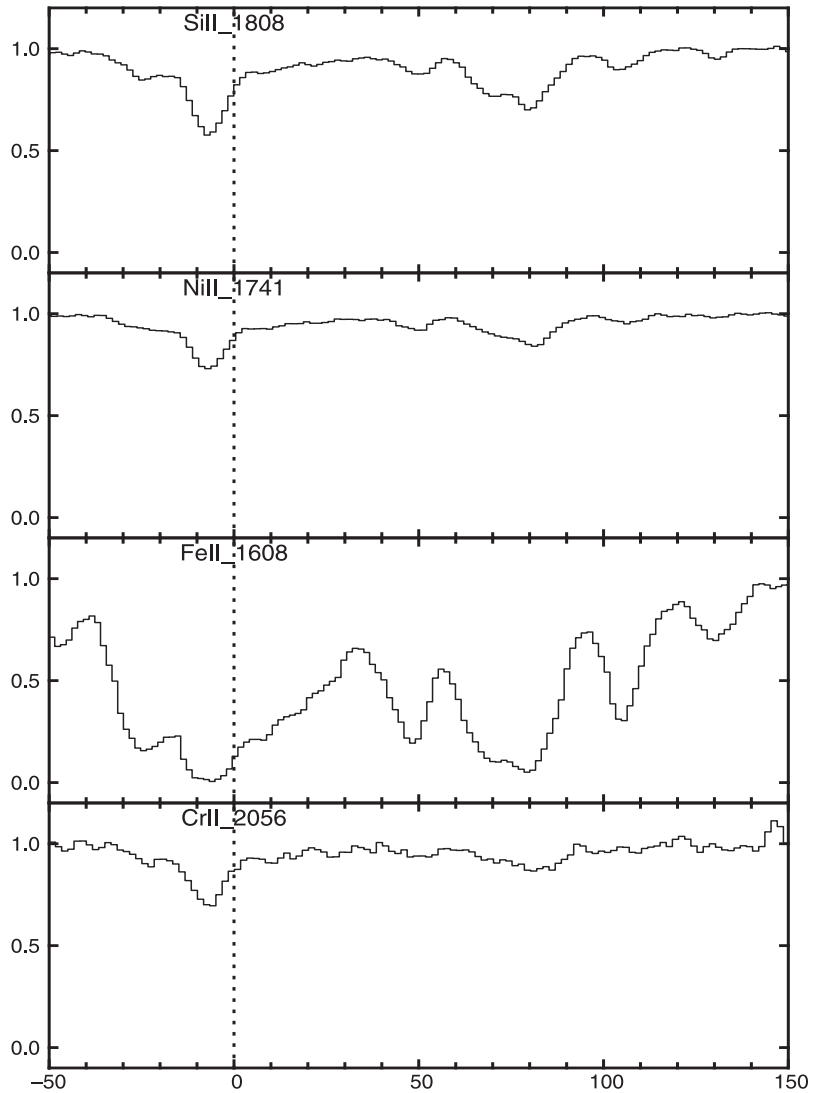

Fig. 3. Absorption spectra plotted on a velocity scale for a few transitions observed in the $z_{\mathrm{abs}}=2.55$ DLA system toward Q 0405-443. (Ledoux et al. 2003)

were detected and the metallicity is the smallest in the sample $([\mathrm{Zn} / \mathrm{H}]=-1.41$, see Table 1$)$.

\section{Analysis method}

We want to estimate the column density per unit velocity along the absorption profile of species $\mathrm{X}$ using several transitions. Following Savage \& Sembach (1991), we can write that the apparent optical depth per unit velocity of species $X$ in the QSO spectrum at wavelength $\lambda$ for any transition of oscillator strength $f$ and rest wavelength $\lambda_{0}$ is $\tau_{\mathrm{a}}(\lambda)=\operatorname{Ln}(1 / F(\lambda))$, and the column density per unit velocity is $\log N_{\mathrm{a}}(\lambda)=\log \tau_{\mathrm{a}}(\lambda)-$ $\log f \lambda_{0}-14.976$. Given a set of $m$ transitions of the same species $\mathrm{X}$, we can use the duplication of the information over the different transition lines to derive the optical depth profile of the species watching out in addition, for possible blending. Let $\lambda_{0}^{k}$ and $f^{k}$ be, respectively, the laboratory wavelength and the oscillator strength of transition $k$. First, the regions corresponding to these transitions are rebinned to the smallest pixel size so that they span the same redshift range over an identical number $n$ of pixels. Let $\lambda_{i}^{k}, F_{i}^{k}$ be the wavelength and the normalized flux at pixel $\mathrm{i}$ of the region corresponding to transition $k$. The value $\lambda_{i}^{k} / \lambda_{0}^{k}-1$ is independent of $k$, thanks to the rebin, and is the redshift at pixel $i$. The observed optical depth, $-\ln F_{i}^{k}$, is considered as the sum of the optical depth, $\tau_{i}^{X k}$, of transition $k$ and the optical depth, $\tau_{i}^{B k}$, of a possible intervening absorption blended with the absorption of interest (of course in

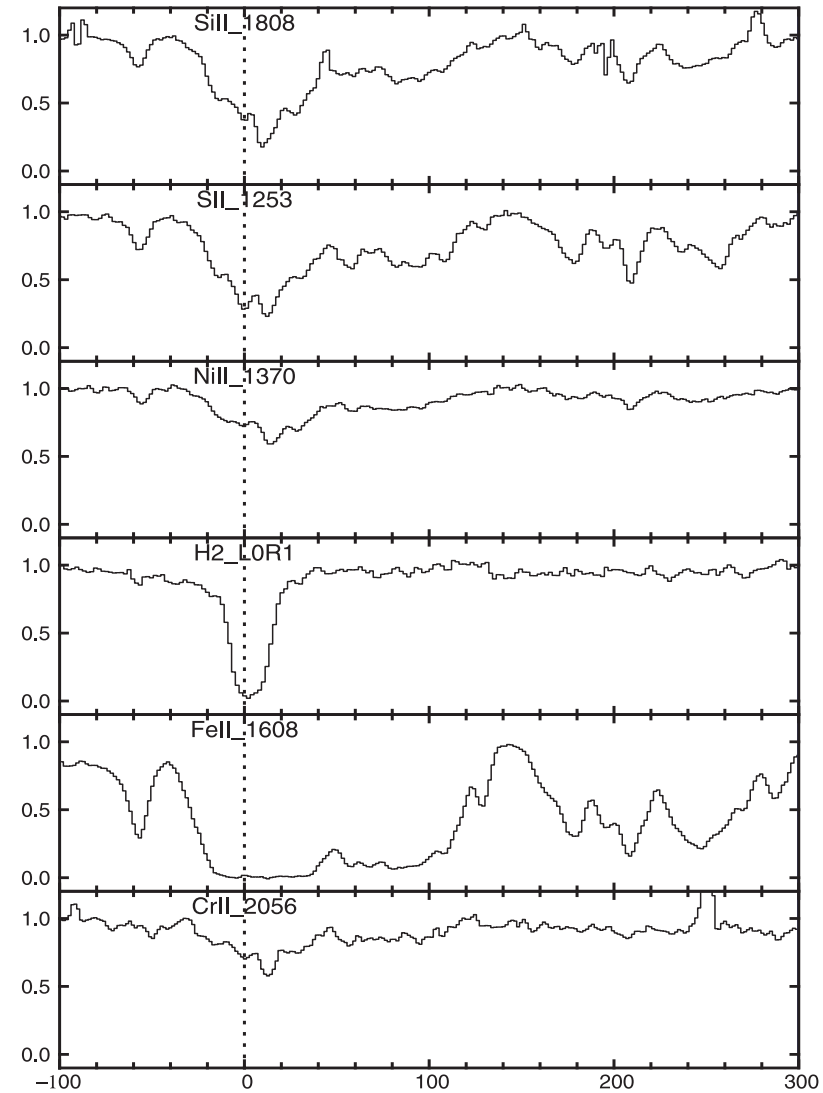

Fig. 4. Absorption spectra plotted on a velocity scale for a few low-ionization species - including H2L0R1 transition - observed at $z_{\text {abs }}=2.811$ along the line of sight to PKS 0528-250. The system extends over more than $400 \mathrm{~km} \mathrm{~s}^{-1}$.

practice we will choose profiles that are apparently not blended with interlopers). As the different transitions are from the same species, the quantity $\log N_{\mathrm{a}}^{k}$ (or simply $\tau_{i}^{X k} / f^{k} \lambda_{0}^{k}$ ) is the same for all transitions $k$.

The fitted optical depth before any instrumental convolution and without adjusting for any overlapping between regions, is then:

$\tau_{i}^{k}=\tau_{i}^{X k}+\tau_{i}^{B k} \quad \forall i \in\{1, \ldots, n\}, \forall k \in\{1, \ldots, m\}$.

To simplify the notation, we use the data vector $\boldsymbol{O}$ of $m * n$ elements, obtained by stacking the observed flux of each region (i.e. $\left.F_{i}^{k}=\boldsymbol{O}_{i+k n-n}\right)$, the parameter vector $\boldsymbol{P}$ of $n+m * n$ elements, obtained by stacking $\tau_{i}^{X k} / f^{k} \lambda_{0}^{k}$ and $\tau_{i}^{B k}$ (i.e. $\tau_{i}^{X k} / f^{k} \lambda_{0}^{k}=\boldsymbol{P}_{i}$ and $\left.\tau_{i}^{B k}=\boldsymbol{P}_{k n+i}\right)$, and the fitted vector $\boldsymbol{F}$ corresponding to the fitted flux. The $\chi^{2}$ of the fit is then:

$$
\chi^{2}=\operatorname{tr}\left([\boldsymbol{W} \cdot(\boldsymbol{O}-\boldsymbol{F})]^{\mathrm{T}} \cdot[\boldsymbol{W} \cdot(\boldsymbol{O}-\boldsymbol{F})]\right)
$$

with $\boldsymbol{F}=\boldsymbol{C} \cdot \exp ^{*}(-\boldsymbol{M} \cdot \boldsymbol{P})$.

The matrix $\boldsymbol{M}$ applies the linear relation (1) to the parameters and takes care of possible overlap between different regions. The function $\exp ^{*}$ replaces each element of a matrix by its exponential value (i.e. $\exp ^{*}(X)_{i j}=\mathrm{e}^{X_{i j}}$ ). The matrix $C$ is used to convolve the calculated flux with the instrumental profile and, finally, $\boldsymbol{W}$ is the inverse of the variance-covariance matrix of the data. 


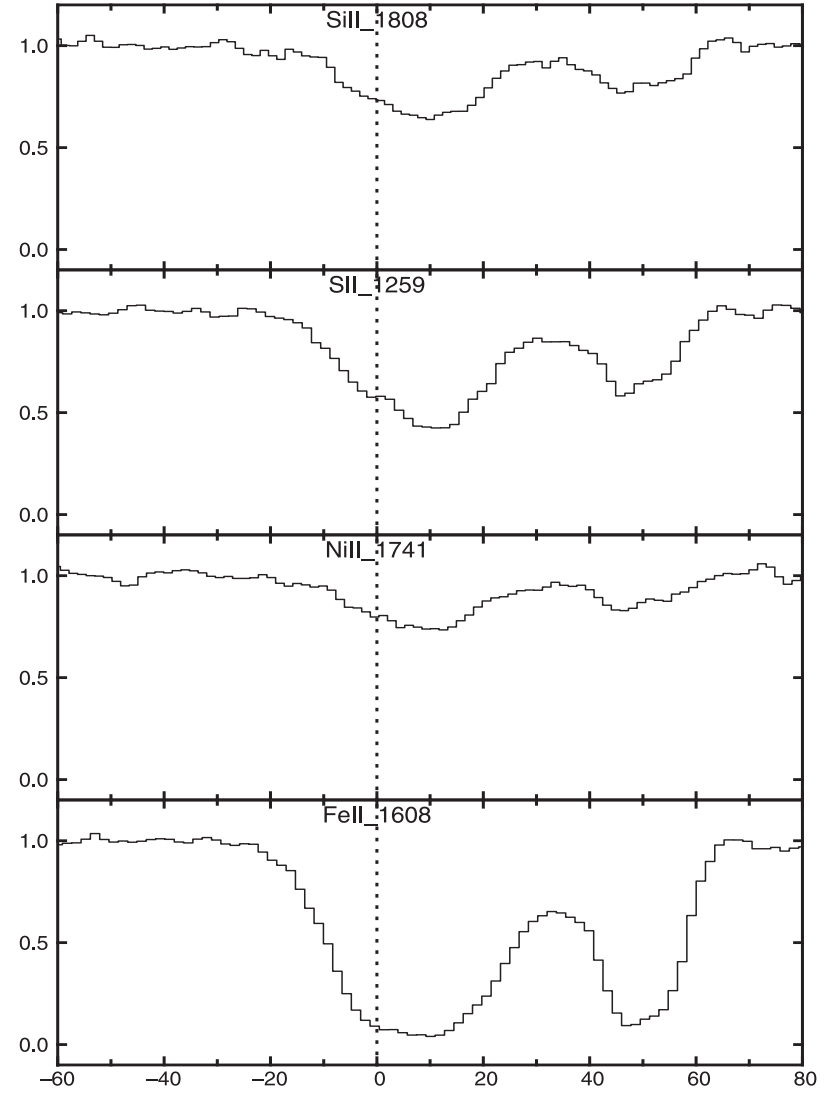

Fig. 5. Absorption spectra plotted on a velocity scale for selected transition lines in the DLA system at $z_{\mathrm{abs}}=2.139$ towards Q 1037-270. The system shows a "smooth" profile for all the elements spread over nearly $100 \mathrm{~km} \mathrm{~s}^{-1}$.

A regularization constraint is added to select the solutions $\boldsymbol{P}$ that are correlated over a specific length $l_{0}$. The selection is done by a minimization of the high frequency coefficients of the discrete Fourier transform of the parameters $\boldsymbol{P}$, i.e. by minimizing $\zeta^{2}$ (Eq. (4) below) at the same time as $\chi^{2}$ (Eq. (2)).

$\zeta^{2}=\operatorname{tr}\left(\left[\boldsymbol{S}_{l_{0}} \cdot \boldsymbol{T} \cdot \boldsymbol{P}\right]^{\mathrm{T}} \cdot\left[\boldsymbol{S}_{l_{0}} \cdot \boldsymbol{T} \cdot \boldsymbol{P}\right]\right)$,

where $\boldsymbol{T}$ is the matrix of the discrete Fourier transform and $\boldsymbol{S}_{l_{0}}$ the high frequency filter that cancels the coefficients of the Fourier transform corresponding to a length (in the parameter space) greater than $l_{0}$.

Actually, the overall fit with the regularization constraint is done by using a Lagrange parameter $f_{0}$ and minimizing the following quantity,

$Q=\chi^{2}+f_{0} \zeta^{2}$.

The parameter $f_{0}$ controls the relative strength of the regularisation over the $\chi^{2}$ fit of the data. If $f_{0}$ is too small, then only $\chi^{2}$ is minimized and the data will be over-fitted. In contrast, if $f_{0}$ is too large, the minimization is done only for $\zeta^{2}$ to give a very smooth solution that does not fit the data. Thus, $f_{0}$ is chosen to obtain a $\chi^{2}$ value approximately equal to 1 . In the worst case, since there are more parameters $(n *(m+1))$ than data points $(n * m)$, the problem is underestimated. Nevertheless, the



Fig. 6. Absorption spectra plotted on a velocity scale for a few transitions observed in the DLA system at $z_{\mathrm{abs}}=1.944$ towards Q $1157+014$.

regularization introduces a constraint that diminishes the real number of free parameters. Moreover, in practice, most of the profiles we use here are free of blending and, in that case, we impose a zero optical depth for the intervening absorption.

Once the different profiles have been fitted, we compute the ratios between the most important elements in order to analyze possible variations of the ratios along the profile. The method is well-suited to studying the complex absorption profiles of DLAs. Indeed, it is well-known that the decomposition in discrete components is not unique due to the fact that the observed absorption profile is a convolution of absorptions from density fluctuations in a continuous medium. In addition, absorptions from different overdensities can be superimposed by peculiar kinematics, making any decomposition in components an ad hoc representation of reality. The pixel-by-pixel method instead does not assume any model and emphasizes deviations from the mean value when a fit tends to smooth such deviations out. Although the mean depletion measured by both methods, the pixel-by-pixel analysis and the sub-component fitting, is approximatively the same, deviations from the mean and in particular locations within the gas, can only be revealed by a pixelby-pixel analysis. In particular, the scatter in the data points is a good estimate of the (in)homogeneity of the gas. 
Table 1. Description of the sample. The neutral and molecular hydrogen column densities were taken from Ledoux et al. (2003). Both [Fe/H] and metallicity values were from this work and correspond to the values integrated over the systems. Metallicity was derived in all cases from [S/H], except for $Q 1157+014$ for which $[\mathrm{S} / \mathrm{H}]$ determination was uncertain, so we used $[\mathrm{Zn} / \mathrm{H}]$ instead.

\begin{tabular}{lcccccc}
\hline \hline Quasar & $z_{\mathrm{em}}$ & $z_{\mathrm{abs}}$ & $\log N(\mathrm{H} \mathrm{I})^{a}$ & $\log N\left(\mathrm{H}_{2}\right)$ & {$[\mathrm{Fe} / \mathrm{H}]$} & Metallicity \\
\hline $0013-004$ & 2.09 & 1.973 & 20.83 & 17.72 & $-1.46 \pm 0.01$ & $-0.68 \pm 0.03$ \\
$0405-443$ & 3.00 & 2.595 & 20.90 & 18.16 & $-1.30 \pm 0.10$ & $-0.93 \pm 0.02$ \\
$0405-443$ & 3.00 & 2.549 & 21.00 & $<13.9$ & $-1.49 \pm 0.01$ & $-0.96 \pm 0.04$ \\
$0528-250$ & 2.80 & 2.811 & 21.35 & 18.22 & $-1.44 \pm 0.01$ & $-1.07 \pm 0.01$ \\
$1037-270$ & 2.31 & 2.139 & 19.70 & $<13.7$ & $-0.51 \pm 0.03$ & $-0.19 \pm 0.02$ \\
$1157+014$ & 1.99 & 1.944 & 21.80 & $<14.5$ & $-1.84 \pm 0.03$ & $-1.41 \pm 0.02$ \\
\hline
\end{tabular}

Table 2. Atomic data.

\begin{tabular}{llll}
\hline \hline Transition & $\begin{array}{l}\lambda_{\text {vac }} \\
\text { (A) }\end{array}$ & $f$ & Ref. \\
\hline Si II $\lambda 1020$ & 1020.6989 & 0.01680 & $\mathrm{a}$ \\
Si II $\lambda 1190$ & 1190.4158 & 0.25000 & $\mathrm{a}$ \\
Si II $\lambda 1304$ & 1304.3702 & 0.08630 & $\mathrm{a}$ \\
Si II $\lambda 1526$ & 1526.7070 & 0.12700 & $\mathrm{a}$ \\
Si II $\lambda 1808$ & 1808.0129 & 0.00208 & $\mathrm{a}$ \\
S II $\lambda 1250$ & 1250.5840 & 0.00545 & $\mathrm{a}$ \\
S II $\lambda 1253$ & 1253.8110 & 0.01090 & $\mathrm{a}$ \\
S II $\lambda 1259$ & 1259.5190 & 0.01620 & $\mathrm{a}$ \\
Cr II $\lambda 2056$ & 2056.2690 & 0.1050 & $\mathrm{a}$ \\
Cr II $\lambda 2062$ & 2062.2361 & 0.0780 & $\mathrm{a}$ \\
Cr II $\lambda 2066$ & 2066.1640 & 0.0515 & $\mathrm{a}$ \\
Fe II $\lambda 1143$ & 1143.2260 & 0.01770 & $\mathrm{a}$ \\
Fe II $\lambda 1144$ & 1144.9379 & 0.10600 & $\mathrm{a}$ \\
Fe II $\lambda 1608$ & 1608.4508 & 0.05800 & $\mathrm{a}$ \\
Fe II $\lambda 1611$ & 1611.2003 & 0.00136 & $\mathrm{a}$ \\
Fe II $\lambda 2249$ & 2249.8768 & 0.00182 & $\mathrm{a}$ \\
Fe II $\lambda 2260$ & 2260.7805 & 0.00244 & $\mathrm{a}$ \\
Fe II $\lambda 2344$ & 2344.2130 & 0.11400 & $\mathrm{a}$ \\
Fe II $\lambda 2374$ & 2374.4603 & 0.0313 & $\mathrm{a}$ \\
Ni II $\lambda 1370$ & 1370.1320 & 0.0765 & $\mathrm{~b}$ \\
Ni II $\lambda 1454$ & 1454.8420 & 0.0300 & $\mathrm{~b}$ \\
Ni II $\lambda 1709$ & 1709.6042 & 0.0324 & $\mathrm{~b}$ \\
Ni II $\lambda 1741$ & 1741.5531 & 0.0427 & $\mathrm{~b}$ \\
Ni II $\lambda 1751$ & 1751.9157 & 0.0277 & $\mathrm{~b}$ \\
Zn II $\lambda 2026$ & 2026.1371 & 0.489 & $\mathrm{a}$ \\
Zn II $\lambda 2062$ & 2062.6604 & 0.256 & $\mathrm{a}$ \\
\hline VacIut & &
\end{tabular}

References: Vacuum wavelengths from Morton (2003).

Oscillator strengths: a) Morton (2003); b) Fedchak et al. (2000).

\section{Results for individual objects}

Several of the systems in the sample are spread over more than $200 \mathrm{~km} \mathrm{~s}^{-1}$. The absorption profiles are often clearly structured in several well-detached clumps with no or very little absorption inbetween. To investigate whether the properties differ from one clump to the other, we performed the analysis on each of the clumps. Indeed, it is reasonable to believe that different part of the DLAs could have different characteristics (e.g. Haehnelt et al. 1998). Column densities of several species, integrated over the system, are given for each system in Table 4. Abundance ratios relative to solar are defined as $[X / Y] \equiv \log [N(X) / N(Y)]-\log [N(X) / N(Y)]_{\odot}$, adopting solar abundances from Anders \& Grevesse (1993). These solar abundances and typical galactic depletion values are
Table 3. Solar abundances and typical galactic depletion values of elements investigated in this paper.

\begin{tabular}{lcccc}
\hline \hline Ion & $\log (X / \mathrm{H})+12^{a}$ & \multicolumn{3}{c}{$[X / \mathrm{S}]^{b, c}$} \\
& & Cold disc & Warm disc & Halo \\
\hline H I & 12.00 & $\ldots$ & $\ldots$ & $\ldots$ \\
Si II & $7.55 \pm 0.02$ & -1.3 & -0.4 & -0.3 \\
S II & $7.27 \pm 0.05$ & +0.0 & +0.0 & +0.0 \\
Cr II & $5.68 \pm 0.03$ & -2.1 & -1.2 & -0.6 \\
Mn II & $5.53 \pm 0.04$ & -1.5 & -1.0 & -0.7 \\
Fe II & $7.51 \pm 0.01$ & -2.2 & -1.4 & -0.6 \\
Ni II & $6.25 \pm 0.02$ & -2.2 & -1.4 & -0.6 \\
Zn II & $4.65 \pm 0.02$ & -0.4 & -0.2 & -0.1 \\
\hline
\end{tabular}

${ }^{a}$ Solar and Meteorite abundances from Anders (1993).

${ }^{b}[X / \mathrm{H}]=\log [Z(X)]-\log \left[Z_{\odot}(X)\right]$.

${ }^{c}$ Galactic values are from Welty et al. (1999).

summarized in Table 3 . We computed the quantity $[X / Y]$ in each pixel and its average $(\overline{[X / Y]})$ over each subclump, and results are given in Table 5. Each subclump is referred to by the velocity range over which it is spread relative to the main redshift of the system, with a reference $z_{\text {abs }}$ taken from (Ledoux et al. 2003). Then $\overline{[X / Y]}$, averaged over this velocity range, is given for each ratio. In each row, the number next to $\overline{[X / Y]}$ is the error on the mean ratio $\left(\sigma=\sqrt{\sum_{i=1}^{n} \sigma_{i}^{2} / n}\right)$, and the second number (in italics) is the scatter of $[X / Y]$ around the mean calculated over the subclump, $\left[\sum\left(\overline{[X / Y]}-[X / Y]_{i}\right)^{2} / n\right]^{1 / 2}$.

\section{1. $Q 0013-004$}

As mentioned in Sect. 2.1, the absorption system found in the line of sight of Q 0013-004 at $z_{\mathrm{abs}}=1.973$ shows a complex multicomponent structure. Besides the fact that the system is composed of a DLA at $z_{\mathrm{abs}}=1.96753$ and a sub-DLA at $z_{\text {abs }}=1.9733$ (Petitjean et al. 2002), the low-ionization absorptions span about $1000 \mathrm{~km} \mathrm{~s}^{-1}$, and the system exhibits four molecular components at $\sim 615,-480,0$ and $85 \mathrm{~km} \mathrm{~s}^{-1}$ relative to the central redshift. Figure 7 illustrates this complexity. The apparent column density per velocity bin, referred to solar values, is plotted for Fe II, S II, and Si II. All have complex but remarkably similar profiles. The bottom panels represent the ratio per velocity bin $[\mathrm{Fe} / \mathrm{S}]$ for different velocity ranges. The mean values (see Table 5) are smaller than in other systems but are again quite similar from one sub-clump to the other. Within a peculiar sub-clump, scatter is small, on the order of 
Table 4. Total column densities and integrated abundance ratios relative to solar in the six DLAs of our sample.

\begin{tabular}{|c|c|c|c|c|c|c|c|}
\hline Species & $N\left(\mathrm{~cm}^{-2}\right)$ & {$[X / S]$} & {$[X / \mathrm{Fe}]$} & Species & $N\left(\mathrm{~cm}^{-2}\right)$ & {$[X / S]$} & {$[X / \mathrm{Fe}]$} \\
\hline Q 0013-004 & $z_{\mathrm{abs}}=1.973$ & & & Q $0405-443$ & $z_{\mathrm{abs}}=2.549$ & & \\
\hline FeII & $7.55 \pm 0.01 \times 10^{14}$ & $-0.78 \pm 0.03$ & $0.00 \pm 0.00$ & FeII & $1.05 \pm 0.01 \times 10^{15}$ & $-0.53 \pm 0.04$ & $0.00 \pm 0.00$ \\
\hline SII & $2.64 \pm 0.08 \times 10^{15}$ & $0.00 \pm 0.00$ & $0.78 \pm 0.03$ & SII & $2.03 \pm 0.07 \times 10^{15}$ & $0.00 \pm 0.00$ & $0.53 \pm 0.14$ \\
\hline SiII & $2.72 \pm 0.20 \times 10^{15}$ & $-0.27 \pm 0.08$ & $0.51 \pm 0.07$ & SiII & $2.38 \pm 0.32 \times 10^{15}$ & $-0.21 \pm 0.15$ & $0.32 \pm 0.14$ \\
\hline $\mathrm{ZnII}$ & $7.30 \pm 1.12 \times 10^{12}$ & $0.06 \pm 0.15$ & $0.84 \pm 0.15$ & CrII & $2.46 \pm 0.76 \times 10^{13}$ & $-0.33 \pm 0.31$ & $0.20 \pm 0.31$ \\
\hline NiII & $7.57 \pm 0.72 \times 10^{13}$ & $-0.52 \pm 0.05$ & $0.26 \pm 0.04$ & NiII & $7.05 \pm 0.96 \times 10^{13}$ & $-0.44 \pm 0.14$ & $0.09 \pm 0.14$ \\
\hline Q 0405-443 & $z_{\mathrm{abs}}=2.595$ & & & Q 0528-250 & $z_{\mathrm{abs}}=2.811$ & & \\
\hline FeII & $1.30 \pm 0.13 \times 10^{15}$ & $-0.37 \pm 0.10$ & $0.00 \pm 0.00$ & FeII & $2.60 \pm 0.03 \times 10^{15}$ & $-0.37 \pm 0.01$ & $0.00 \pm 0.00$ \\
\hline SII & $1.75 \pm 0.04 \times 10^{15}$ & $0.00 \pm 0.00$ & $0.37 \pm 0.10$ & SII & $3.49 \pm 0.02 \times 10^{15}$ & $0.00 \pm 0.00$ & $0.37 \pm 0.01$ \\
\hline SiII & $3.52 \pm 0.07 \times 10^{15}$ & $0.03 \pm 0.03$ & $0.40 \pm 0.10$ & SiII & $1.01 \pm 0.05 \times 10^{16}$ & $0.18 \pm 0.05$ & $0.55 \pm 0.05$ \\
\hline CrII & $2.23 \pm 0.07 \times 10^{13}$ & $-0.30 \pm 0.04$ & $0.06 \pm 0.10$ & $\mathrm{ZnII}$ & $1.27 \pm 0.26 \times 10^{13}$ & $0.18 \pm 0.21$ & $0.55 \pm 0.21$ \\
\hline \multirow[t]{2}{*}{ NiII } & $6.92 \pm 0.08 \times 10^{13}$ & $-0.38 \pm 0.02$ & $-0.02 \pm 0.10$ & CrII & $7.18 \pm 1.45 \times 10^{13}$ & $-0.10 \pm 0.20$ & $0.17 \pm 0.20$ \\
\hline & & & & NiII & $1.62 \pm 0.32 \times 10^{14}$ & $-0.31 \pm 0.20$ & $0.05 \pm 0.20$ \\
\hline Q 1037-270 & $z_{\mathrm{abs}}=2.139$ & & & Q $1157+014$ & $z_{\mathrm{abs}}=1.944$ & {$[X / \mathrm{Zn}]$} & \\
\hline FeII & $4.98 \pm 0.14 \times 10^{14}$ & $-0.32 \pm 0.04$ & $0.00 \pm 0.00$ & FeII & $2.95 \pm 0.08 \times 10^{15}$ & $-0.43 \pm 0.04$ & $0.00 \pm 0.00$ \\
\hline SII & $6.05 \pm 0.15 \times 10^{14}$ & $0.00 \pm 0.00$ & $0.32 \pm 0.04$ & SilI & $1.00 \pm 0.01 \times 10^{16}$ & $0.06 \pm 0.02$ & $0.50 \pm 0.03$ \\
\hline SilI & $1.89 \pm 0.15 \times 10^{15}$ & $0.21 \pm 0.08$ & $0.53 \pm 0.09$ & ZnII & $1.09 \pm 0.02 \times 10^{13}$ & $0.00 \pm 0.00$ & $0.43 \pm 0.04$ \\
\hline CrII & $1.02 \pm 0.58 \times 10^{13}$ & $-0.18 \pm 0.57$ & $0.14 \pm 0.57$ & CrII & $6.07 \pm 0.19 \times 10^{13}$ & $-0.29 \pm 0.04$ & $0.14 \pm 0.04$ \\
\hline NiII & $6.43 \pm 1.04 \times 10^{13}$ & $0.05 \pm 0.16$ & $0.36 \pm 0.16$ & NiII & $1.62 \pm 0.05 \times 10^{14}$ & $-0.43 \pm 0.04$ & $0.00 \pm 0.04$ \\
\hline MnII & $2.22 \pm 0.08 \times 10^{12}$ & $-0.70 \pm 0.04$ & $-0.37 \pm 0.05$ & & & & \\
\hline
\end{tabular}

0.2 dex. In the components where $\mathrm{H}_{2}$ is detected, the depletion factor is of the order on $[\mathrm{Fe} / \mathrm{S}]=-1$, therefore slightly smaller but not much less than in the overall system, except in the component at $-480 \mathrm{~km} \mathrm{~s}^{-1}$, which is the only region in DLAs known to date where depletion is similar to the cold gas of the Galactic disc.

\subsection{Q $0405-443$}

We analyzed the systems at $z_{\mathrm{abs}}=2.549$ and $z_{\mathrm{abs}}=2.595$ toward Q 0405-443. In the latter system, molecular hydrogen has been detected with a column density, $\log N\left(\mathrm{H}_{2}\right)=18.16$ (Ledoux et al. 2003). Relatively narrow, low-ionization transitions span about $70 \mathrm{~km} \mathrm{~s}^{-1}$. It is apparent from Fig. 10 that there are some inhomogeneities inside the system and there is more depletion (by about a factor of two) in the two components where $\mathrm{H}_{2}$ is detected at $v \sim-8$ and $-24 \mathrm{~km} \mathrm{~s}^{-1}$. Note that the depletion is larger in these components, but not by a large amount and definitively not as in cold gas of the Galaxy disc. The scatter of the pixel values is much larger than the errors for all element ratios (see Table 5). The overall depletion pattern is again similar to that of warm gas of the halo, with a slight enhancement of $\mathrm{Cr}$.
The system at $z_{\mathrm{abs}}=2.549$, presented in Fig. 9, shows absorptions spread over $\sim 120 \mathrm{~km} \mathrm{~s}^{-1}$, and is well-structured in three subclumps. From both Fig. 9 and Table 5, it can be seen that the three subclumps at velocities [-30, 20], [40, 60], and $[60,90]$ are quite homogeneous but have slightly different mean depletion factors, $[\mathrm{Fe} / \mathrm{S}]=-0.26,-0.08$, and -0.33 , respectively. However again, the differences are smaller than 0.2 dex. In addition, the depletion values are close to those of the $z_{\mathrm{abs}}=2.595$ system, which is located $\sim-3000 \mathrm{~km} \mathrm{~s}^{-1}$ away.

\section{3. $Q 0528-250$}

This system is spread over more than $350 \mathrm{~km} \mathrm{~s}^{-1}$ and exhibits two molecular components located at $\sim 0 \mathrm{~km} \mathrm{~s}^{-1}$. We observe in Fig. 11 that the internal structure is complex with many components. Note that the profiles of $\mathrm{Fe}$ and $\mathrm{S}$ follow each other remarkably well over most of the profile. The analysis along the profile gives similar results for each of the subclumps, suggesting that once again the mixing of heavy element must have been very efficient in this system (see Table 5). The central part at $[0,20]$ has a larger depletion coefficient, down to $[\mathrm{Fe} / \mathrm{S}]=-0.55$, when the rest of the system has $[\mathrm{Fe} / \mathrm{S}] \sim-0.3$. 
Table 5. This table summarizes the abundance ratios for the six systems studied. Each system was decomposed in different sub-clumps. For each range of velocities, we determined the mean value of the $[X / \mathrm{S}]$ ratio, its error $\sigma=\sqrt{\sum_{i=1}^{n} \sigma_{i}^{2} / n}$, as well as the standard deviation around the mean (in italics), as an indicator of the inhomogeneity through the subclump. The molecular components were isolated and their depletion values marked in bold face.

\begin{tabular}{|c|c|c|c|c|c|}
\hline System & Sub-clump $\left[\mathrm{km} \mathrm{s}^{-1}\right]$ & {$[\mathrm{Fe} / \mathrm{S}]$} & {$[\mathrm{Si} / \mathrm{S}]$} & {$[\mathrm{Ni} / \mathrm{S}]$} & {$[\mathrm{Cr} / \mathrm{S}]$} \\
\hline \multirow[t]{8}{*}{ Q 0013-004 } & {$[-650,-500]$} & $-0.92 \pm 0.02 \pm 0.15$ & $-0.55 \pm 0.03 \pm 0.13$ & $-0.48 \pm 0.10 \pm 0.23$ & - \\
\hline & {$[-630,-605]$} & $-1.04 \pm 0.05$ & $-0.62 \pm 0.06$ & $-0.75 \pm 0.25$ & - \\
\hline & {$[-490,-465]$} & $-1.60 \pm 0.06$ & $-1.14 \pm 0.26$ & - & - \\
\hline & {$[-220,-100]$} & $-0.80 \pm 0.02 \pm 0.07$ & $-0.31 \pm 0.08 \pm 0.08$ & - & - \\
\hline & {$[-45,+50]$} & $-0.78 \pm 0.01 \pm 0.20$ & $-0.24 \pm 0.06 \pm 0.22$ & $-0.61 \pm 0.06 \pm 0.18$ & - \\
\hline & {$[-15,+15]$} & $-0.90 \pm 0.01$ & $-0.33 \pm 0.01$ & $-0.73 \pm 0.05$ & - \\
\hline & {$[+50,+100]$} & $-0.61 \pm 0.02 \pm 0.16$ & $-0.24 \pm 0.06 \pm 0.15$ & $-0.36 \pm 0.09 \pm 0.21$ & - \\
\hline & {$[+76,+95]$} & $-0.64 \pm 0.03$ & & $-0.34 \pm 0.04$ & $-\mathbf{0 . 5 0} \pm \mathbf{0 . 1 0}$ \\
\hline Q 0405-443 & {$[-60,+10]$} & $-0.27 \pm 0.02 \pm 0.13$ & $0.07 \pm 0.01 \pm 0.08$ & $-0.29 \pm 0.02 \pm 0.15$ & $-0.16 \pm 0.03 \pm 0.17$ \\
\hline \multirow[t]{2}{*}{$z_{\mathrm{abs}}=2.595$} & {$[-30,-14]$} & $-0.34 \pm 0.01$ & $0.03 \pm 0.01$ & $-0.37 \pm 0.01$ & $-0.16 \pm 0.03$ \\
\hline & {$[-17,-2]$} & $-0.37 \pm 0.01$ & $\mathbf{0 . 0 7} \pm \mathbf{0 . 0 1}$ & $-0.38 \pm 0.01$ & $-0.23 \pm 0.04$ \\
\hline Q 0405-443 & {$[-30,+20]$} & $-0.26 \pm 0.02 \pm 0.08$ & $0.07 \pm 0.02 \pm 0.05$ & $-0.17 \pm 0.04 \pm 0.11$ & $0.05 \pm 0.06 \pm 0.10$ \\
\hline \multirow[t]{2}{*}{$z_{\mathrm{abs}}=2.549$} & {$[+40,+60]$} & $-0.08 \pm 0.08 \pm 0.13$ & $0.17 \pm 0.10 \pm 0.12$ & $-0.05 \pm 0.10 \pm 0.04$ & $0.00 \pm 0.26 \pm 0.18$ \\
\hline & {$[+60,+90]$} & $-0.33 \pm 0.02 \pm 0.04$ & $0.09 \pm 0.02 \pm 0.07$ & $-0.23 \pm 0.04 \pm 0.05$ & $-0.18 \pm 0.08 \pm 0.10$ \\
\hline Q $0528-250$ & {$[-30,+40]$} & $-0.31 \pm 0.01 \pm 0.11$ & $0.27 \pm 0.01 \pm 0.10$ & $-0.25 \pm 0.01 \pm 0.11$ & - \\
\hline \multirow[t]{3}{*}{$z_{\mathrm{abs}}=2.811$} & {$[-8,+8]$} & $-0.44 \pm 0.01$ & $0.18 \pm 0.01$ & $-0.38 \pm 0.02$ & - \\
\hline & {$[+50,+110]$} & $-0.29 \pm 0.02 \pm 0.10$ & $0.14 \pm 0.02 \pm 0.06$ & $-0.27 \pm 0.04 \pm 0.06$ & - \\
\hline & {$[+165,+290]$} & $-0.50 \pm 0.05 \pm 0.20$ & $-0.02 \pm 0.02 \pm 0.12$ & $-0.49 \pm 0.06 \pm 0.17$ & - \\
\hline Q 1037-270 & {$[-10,+30]$} & $-0.34 \pm 0.04 \pm 0.07$ & $0.17 \pm 0.03 \pm 0.03$ & $-0.01 \pm 0.04 \pm 0.04$ & - \\
\hline \multirow[t]{2}{*}{$z_{\mathrm{abs}}=2.139$} & {$[+40,+60]$} & $-0.22 \pm 0.07 \pm 0.02$ & $0.16 \pm 0.05 \pm 0.04$ & $0.00 \pm 0.07 \pm 0.05$ & - \\
\hline & & {$[\mathrm{Fe} / \mathrm{Zn}]$} & {$[\mathrm{Si} / \mathrm{Zn}]$} & {$[\mathrm{Ni} / \mathrm{Zn}]$} & {$[\mathrm{Cr} / \mathrm{Zn}]$} \\
\hline Q 1157+014 & {$[-70,+15]$} & $-0.43 \pm 0.02 \pm 0.12$ & $0.05 \pm 0.02 \pm 0.05$ & $-0.43 \pm 0.03 \pm 0.12$ & $-0.29 \pm 0.02 \pm 0.12$ \\
\hline$z_{\mathrm{abs}}=1.944$ & & & & & \\
\hline
\end{tabular}

This is the place where, again, $\mathrm{H}_{2}$ is detected. Even though the depletion factor is larger in this component, it is much smaller than in the cold gas of the Galactic ISM. The overall depletion pattern in the system is very close to what is observed in the Galactic halo (see Table 5).

\section{4. $Q 1037-270$}

Figure 12 shows the column density per pixel along the profile for different species, including Fe II, Si II, S II, and Ni II. All species follow the same pattern, and their ratios remain fairly constant across the profile.

We considered two subclumps for $-10<v<30$ and $40<v<60 \mathrm{~km} \mathrm{~s}^{-1}$ (see Table 5). There is essentially no difference between the two subclumps. Note that the scatter is comparable to or smaller than the errors (for the first $[-10,30]$ subclump, $\sigma_{\mathrm{e}}=0.04, \sigma_{\mathrm{s}}=0.07$ and for the second, [40, $60], \sigma_{\mathrm{e}}[\mathrm{Fe} / \mathrm{S}]=0.07$ and $\left.\sigma_{\mathrm{s}}=0.02\right)$. This system is therefore fairly homogeneous within $0.1 \mathrm{dex}$, and the abundance pattern similar to what is observed in warm gas of the Galactic halo.

\section{5. $Q 1157+014$}

This system is spread over about $100 \mathrm{~km} \mathrm{~s}^{-1}$ and no associated molecules were detected. We considered only one clump. Pixel analysis is shown in Fig. 13. Unfortunately, S II lines lie at the bottom of a BAL trough, so we used zinc as the non-depleted species reference instead.

The system has a low mean metallicity, $[\mathrm{Zn} / \mathrm{H}]=-1.40$ (see Table 1), and shows a depletion pattern similar to the halo of the galaxy for every element analyzed. Pixel analysis is shown in Fig. 13. It can be seen that depletion is larger, $[\mathrm{Fe} / \mathrm{Zn}] \sim-0.65$, in the strongest absorption component. It should be noticed that $21 \mathrm{~cm}$ absorption has been reported by Kanekar \& Chengalur (2003) at this velocity $\left(\sim-28 \mathrm{~km} \mathrm{~s}^{-1}\right)$, revealing dense gas. The difference in depletion between the strongest subcomponent (at $-28 \mathrm{~km} \mathrm{~s}^{-1}$ ) and the gas at $v \sim-40 \mathrm{~km} \mathrm{~s}^{-1}$ is significant (about a factor of two larger than $3 \sigma$ ). This is clearly a sign of more depletion in the dense gas producing the $21 \mathrm{~cm}$ absorption. The overall scatter is only about 0.12 dex (see Table 5). This is fairly small compared to what is observed through the ISM of our Galaxy (Welty et al. 1999). In addition, Ni and Fe follow each other very well. 


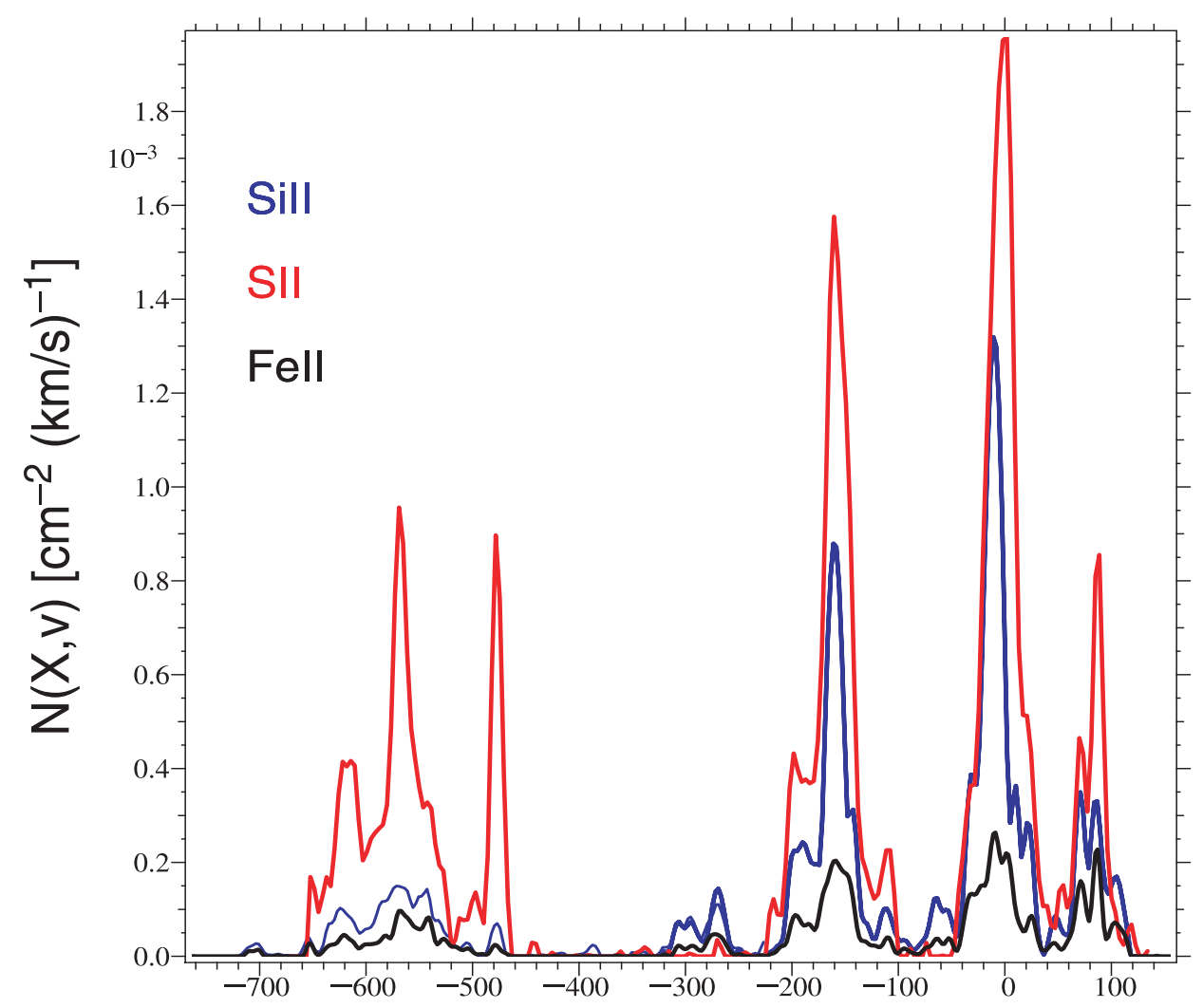

Fig. 7. This panel shows the optical depth profiles in the $z_{\mathrm{abs}}=1.973$ DLA toward Q 0013-004. The apparent column density per velocity bin $-N(X, \mathrm{~d} v)\left(y\right.$ axis values are scaled to $\left.\times 10^{-3}\right)-$ is represented on a velocity scale, with $v=0 \mathrm{~km} \mathrm{~s}^{-1}$ centered at $z_{\text {abs }}=1.973$.

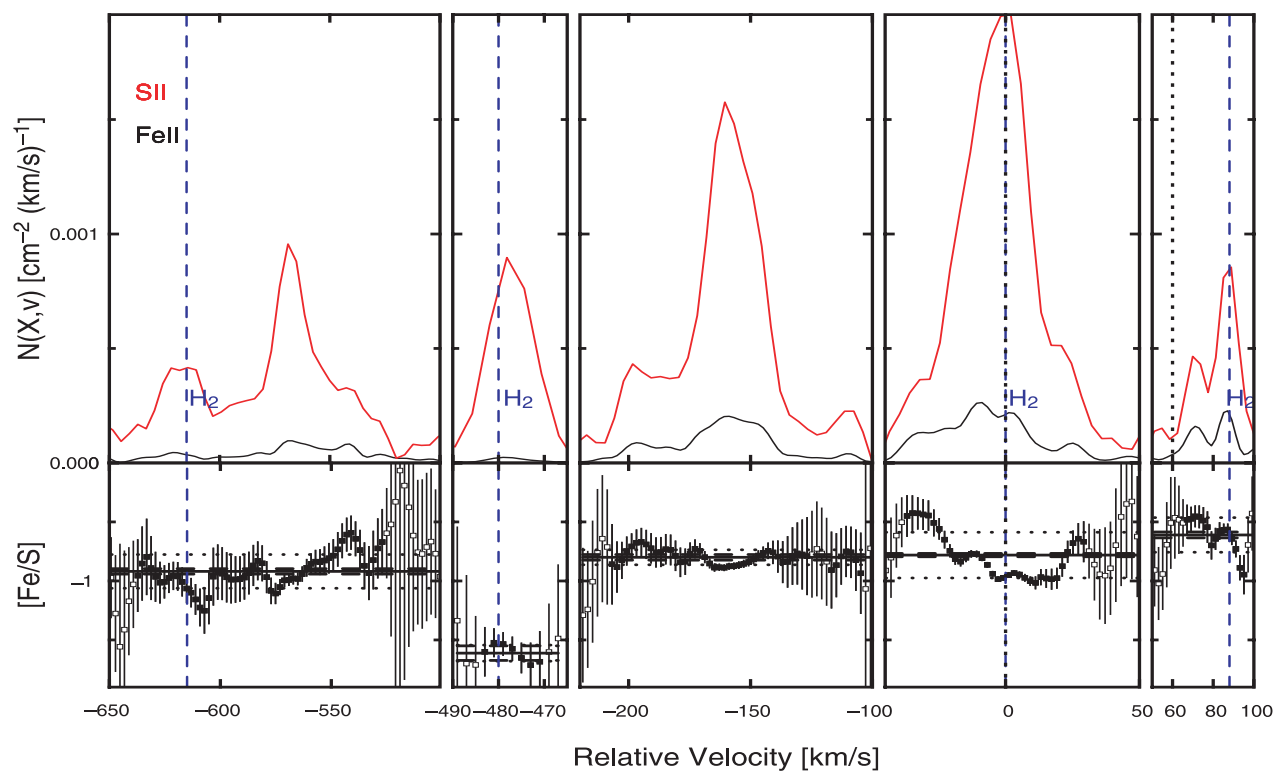

Fig. 8. In this figure, top panels show the same Fe and $\mathrm{S}$ profiles than Fig. 7, divided in five regions. Lower pannels show the ratio [Fe/S] computed for each pixel. Points with errors that are too big are rejected to compute the mean, represented here as empty squares, while valid points are represented as filled squares. The mean value is plotted as a solid line. Dashed lines represent the $\pm \sigma$ level, and the dotted line the typical scatter of the points. $\mathrm{H}_{2}$ is detected in four components at $\sim-615,-480,0$, and $85 \mathrm{~km} \mathrm{~s}^{-1}$.

\section{Discussion}

\subsection{Relative abundance ratios}

Relative abundance ratios for each sub-clump considered in the six systems analyzed in this work are given in Table 5. In the same way, results are summarized in Fig. 14. It is apparent that, within each subsystem, large departures from the mean ratio are rare: the scatter is small if we take the observational and fitting uncertainties into account. 


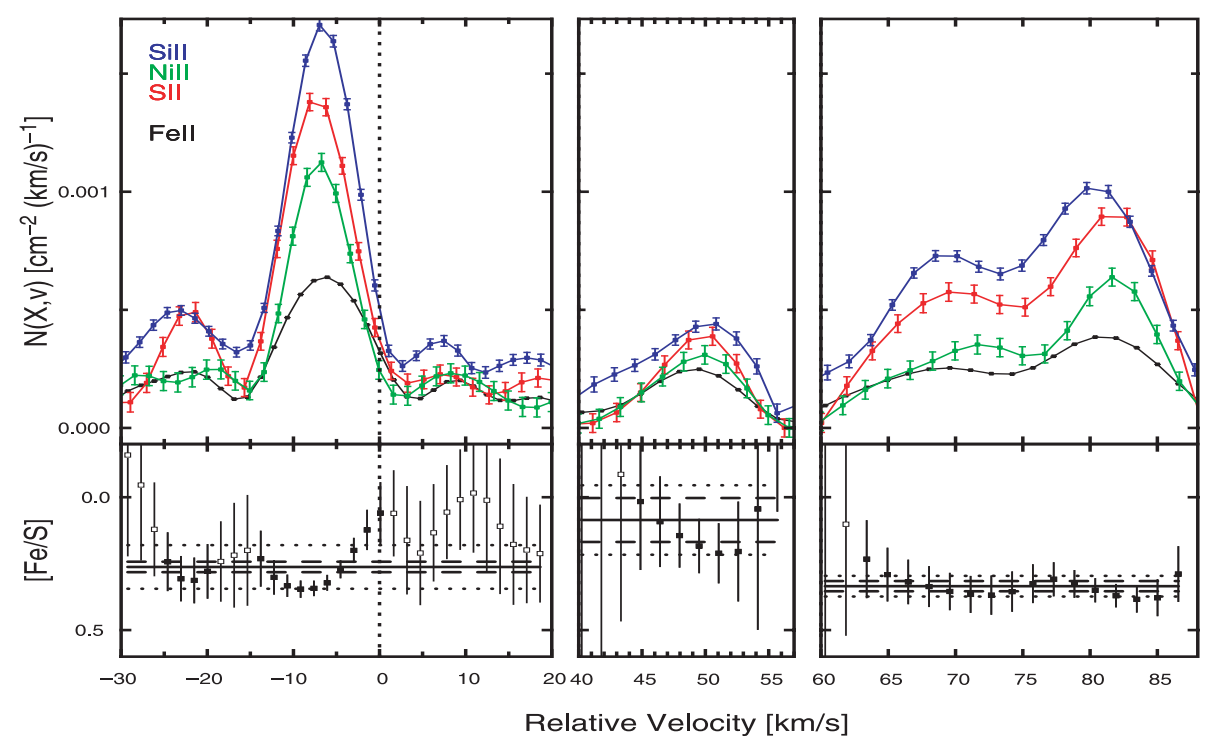

Fig. 9. Top panel: optical depth profiles in the $z_{\mathrm{abs}}=2.549$ DLA toward Q $0405-443$. The apparent column density per velocity bin $-N(X, \mathrm{~d} v)$ - is represented on a velocity scale for $\mathrm{Si}, \mathrm{Ni}, \mathrm{S}$, and $\mathrm{Fe}$; with $v=0 \mathrm{~km} \mathrm{~s}^{-1}$ centered at $z_{\mathrm{abs}}=2.549$. The lower panels show the ratio [Fe/S] computed for each pixel. The mean value is plotted as a solid line. Dashed lines represent the $\pm \sigma$ level and the dotted line the typical scatter of the points.

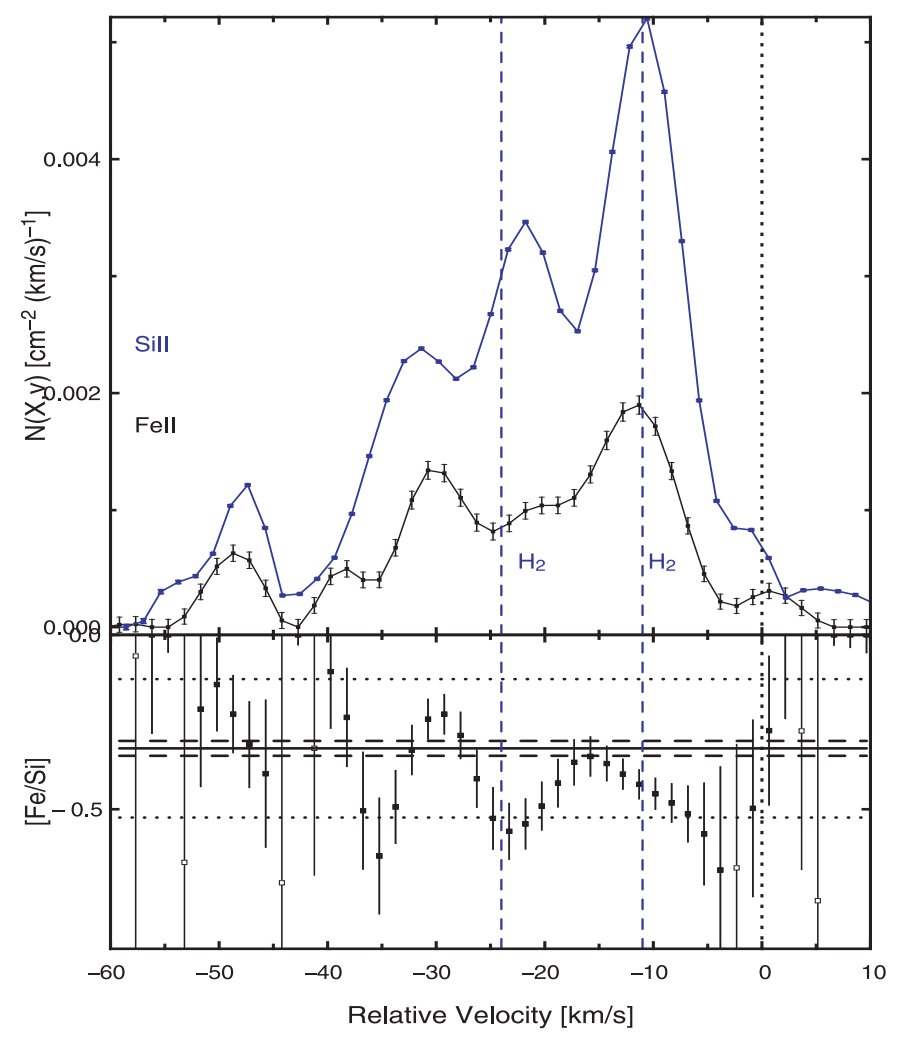

Fig. 10. Top panel: optical depth profiles in the $z_{\text {abs }}=2.595$ DLA toward Q 0405-443. The apparent column density per velocity bin $-N(X, \mathrm{~d} v)-$ is represented on a velocity scale, with $v=0 \mathrm{~km} \mathrm{~s}^{-1}$ centered at $z_{\text {abs }}=2.595$. The lower panels show the ratio $[\mathrm{Fe} / \mathrm{Si}]$ computed for each pixel. The mean value is plotted as a solid line. Dashed lines represent the $\pm \sigma$ level and the dotted line the typical scatter of the points. This DLA has two molecular components at $v \sim-24$ and $\sim-11 \mathrm{~km} \mathrm{~s}^{-1}$.
Moreover, when we compare depletion values from one sub-clump to another in the same system, differences are small. A distinct depletion pattern is observed only for some molecular components .This is remarkably summarized by Fig. 15, where we plot the $[\mathrm{Fe} / \mathrm{S}]$ ratio versus the $[\mathrm{Si} / \mathrm{S}]$ ratio in all subclumps. First, as already emphasized by Petitjean et al. (2002) and Ledoux et al. (2002b), the sequence seen in this figure is a dust-depletion sequence. Indeed, there is a correlation between the two quantities which is expected if the depletion is due to the presence of dust. Secondly, the values measured in different clumps of the same system are gathered at the same place in the figure. The only exception is the $\mathrm{H}_{2}$ component at $-480 \mathrm{~km} \mathrm{~s}^{-1}$ in Q 0013-004 (see above). Thirdly, most of the depletion pattern is similar to that of the gas observed in the Galactic halo. Finally, it seems that silicon is overabundant by about $0.2 \mathrm{dex}$ even relative to sulfur. In all this, however, it must be recalled that we do not have access to the absolute metallicity in the subclumps, because we are not able to disentangle the $\mathrm{H} \mathrm{I}$ absorptions of the different subclumps.

In the left hand panel of Fig. 16, we plot the scatter, measured as $\sigma$, of the ratio $[\mathrm{Fe} / \mathrm{S}]$ in all the subclumps considered. In this case, we considered the subclumps as a whole, not isolating the molecular component, as our aim was to see if there is a relation between the inhomogeneity of a system and its molecular content. The mean value of $\sigma$ over the subclumps is 0.3 , which means that inhomogeneities are less than a factor of 2. Only a few subclumps where $\mathrm{H}_{2}$ is detected have larger $\sigma$. This is expected because we have seen that depletion is larger over the specific small velocity ranges over which $\mathrm{H}_{2}$ is detected.

In the right hand panel of Fig. 16, we plot the different scatter values for each subclump as a function of the total $[\mathrm{Fe} / \mathrm{S}]$ ratio. This figure confirms that (i) larger $[\mathrm{Fe} / \mathrm{S}]$ ratios are 


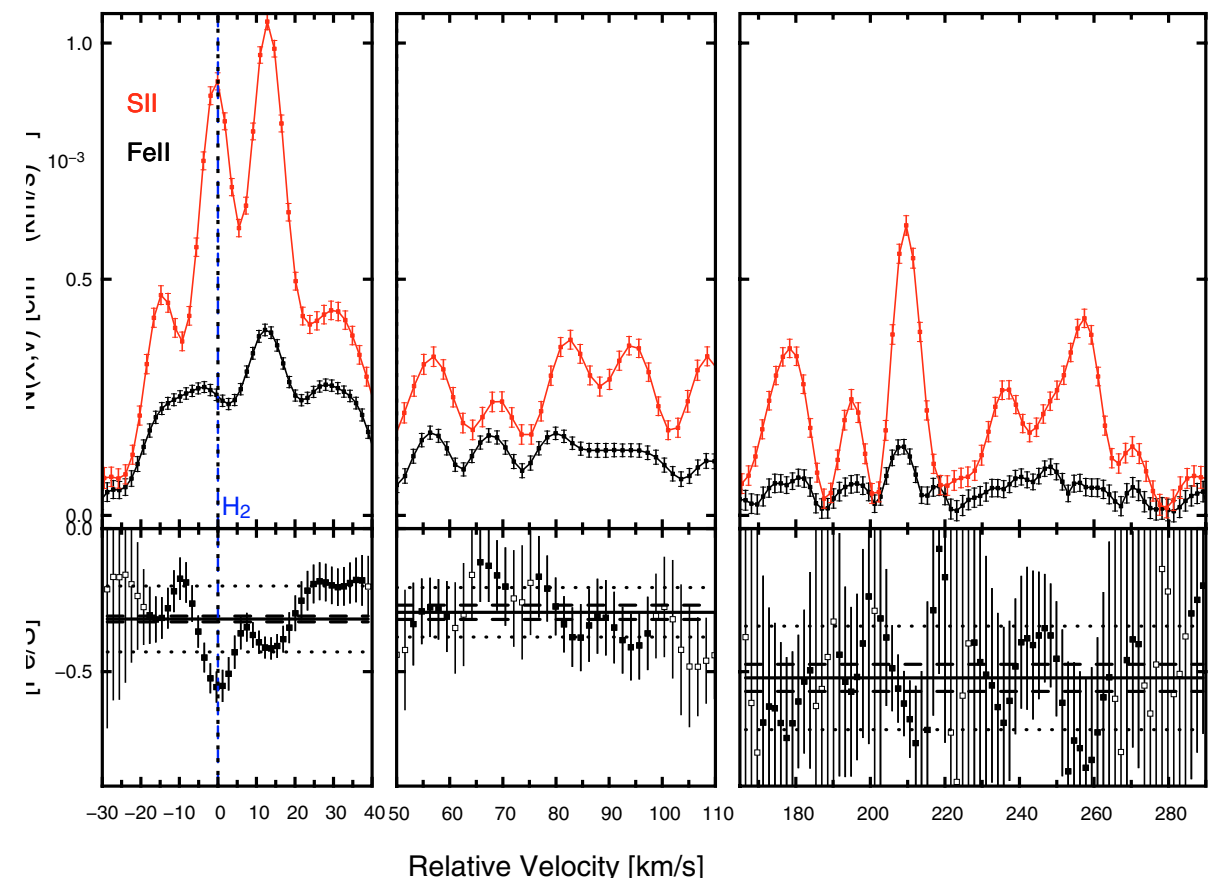

Fig. 11. Top panel: optical depth profiles in the $z_{\mathrm{abs}}=2.811$ DLA toward Q $0528-250$. The apparent column density per velocity bin $-N(X, \mathrm{~d} v)$ - is represented on a velocity scale, with $v=0 \mathrm{~km} \mathrm{~s}^{-1}$ centered at $z_{\mathrm{abs}}=2.811$. The lower panels show the ratio [Fe/S] computed for each pixel. The mean value is plotted as a solid line. Dashed lines represent the $\pm \sigma$ level and the dotted line the typical scatter of the points. $\mathrm{H}_{2}$ is detected in two components at $\sim 0 \mathrm{~km} \mathrm{~s}^{-1}$.

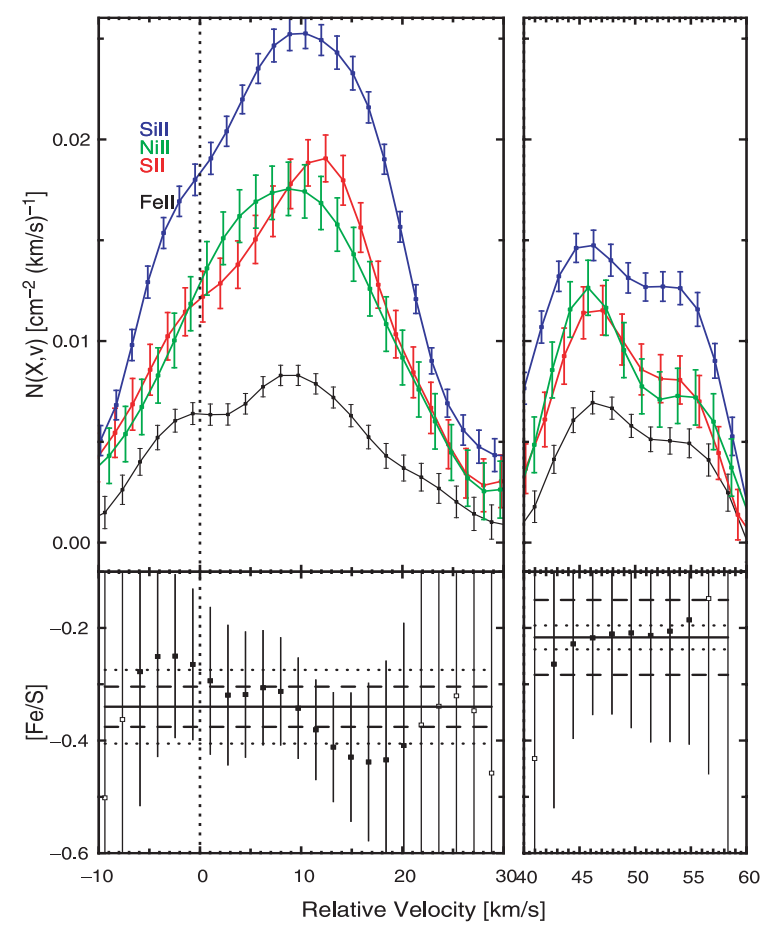

Fig. 12. Top panel: optical depth profiles in the $z_{\text {abs }}=2.139$ DLA towards Q 1037-270. The apparent column density per velocity bin $N(X, \mathrm{~d} v)$ - is represented on a velocity scale, with $v=0 \mathrm{~km} \mathrm{~s}^{-1}$ centered at $z_{\text {abs }}=2.139$. The lower panel shows the ratio $[\mathrm{Fe} / \mathrm{S}]$ computed for each pixel. The mean value is plotted as a solid line. Dashed lines represent the $\pm \sigma$ level and the dotted line the typical scatter of the points.

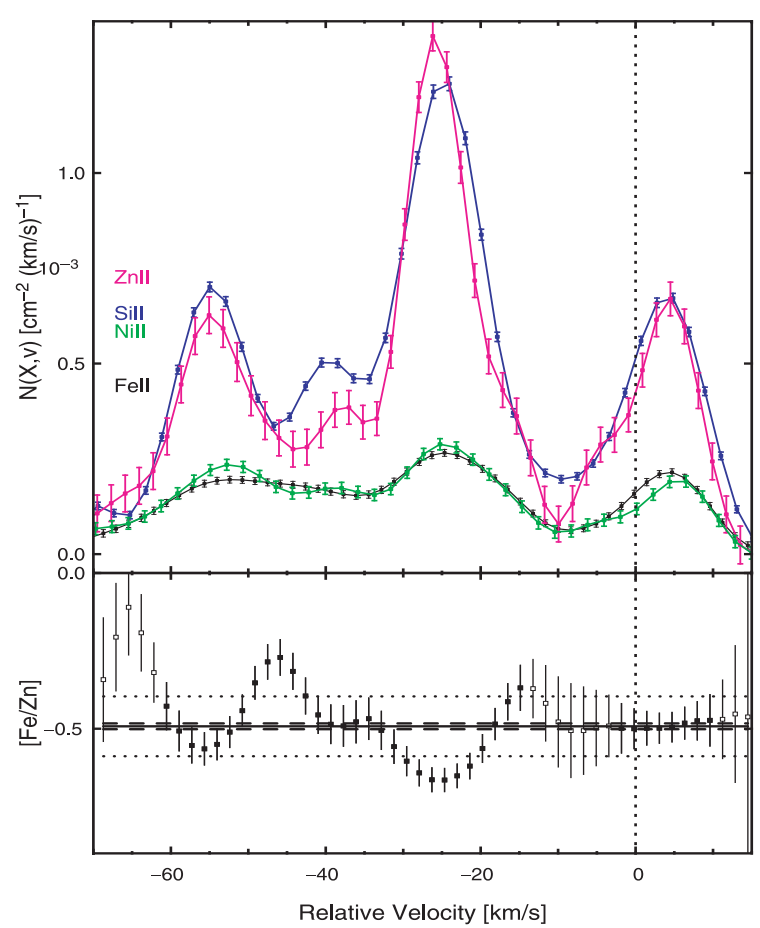

Fig. 13. Top panel: optical depth profiles in the $z_{\text {abs }}=1.944$ DLA towards Q $1157+014$. The apparent column density per velocity bin $-N(X, \mathrm{~d} v)-$ is represented on a velocity scale for $\mathrm{Zn}, \mathrm{Si}, \mathrm{Ni}$, and $\mathrm{Fe}$ species, with $v=0 \mathrm{~km} \mathrm{~s}^{-1}$ centered at $z_{\mathrm{abs}}=1.944$. The lower panel shows the ratios $[\mathrm{Fe} / \mathrm{Zn}]$ computed for each pixel. The mean value is plotted as a solid line. Dashed lines represent the $\pm \sigma$ level, and the dotted line the typical scatter of the points. 

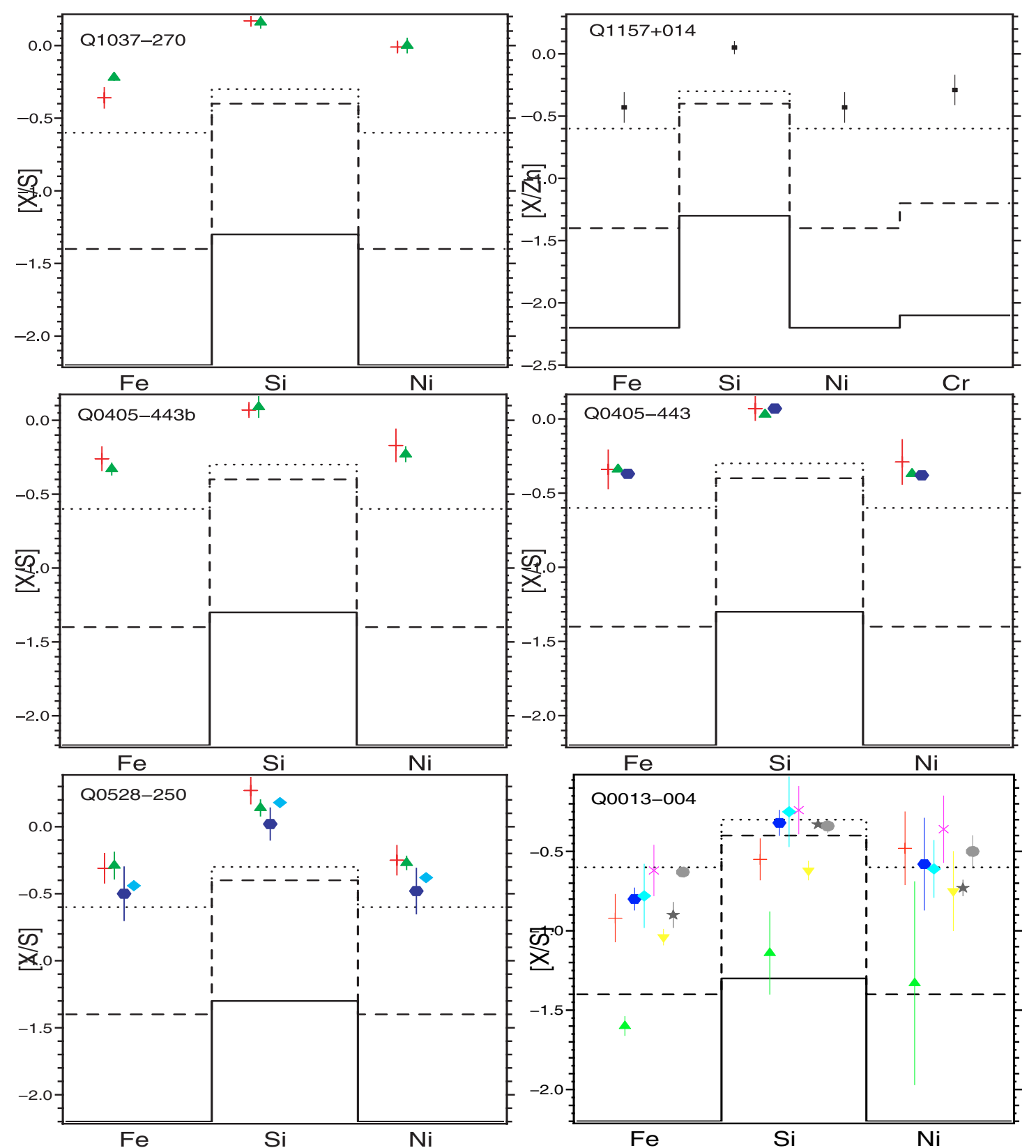

Fig. 14. Depletion of heavy elements relative to Sulfur (except for Q 1157+014, where Zinc was used instead) in the different subclumps of the six DLA systems studied here. Filled symbols represent the different sub-systems considered (see Table 5). Error bars correspond to typical scatter for each sub-system. The histograms show the observed values in the cold (solid line) or warm (dashed line) disc clouds and in halo clouds (dotted line) of the Galaxy.

observed in subclumps where $\mathrm{H}_{2}$ is detected with one exception in a subclump of Q 0013-0004, and that (ii) the scatter is larger for subclumps where $\mathrm{H}_{2}$ is detected.

\subsection{The presence of $\mathrm{H}_{2}$ molecules}

Ledoux et al. (2003) have systematically searched for molecular hydrogen in high redshift DLAs, with a $\sim 20 \%$ detection rate over the whole sample. The observed molecular fraction is often much smaller than in the ISM of the Galactic disc (Rachford et al. 2002) and is closer to what is observed in the Magellanic clouds (Tumlinson et al. 2002). Here, we confirm what was already noticed by Ledoux et al. (2002b) and Petitjean et al. (2002) that, although the presence of molecules sometimes reveals gas with larger depletion into dust grains than average, this is not always the case. In most of the systems, the depletion factor is only a factor of two larger in the components with $\mathrm{H}_{2}$ compared to the overall system. There are a few exceptions, the most noticeable being the molecular component at $-480 \mathrm{~km} \mathrm{~s}^{-1}$ toward Q $0013-014$, in which depletion is as large as in the cold gas of the Galactic disc. 


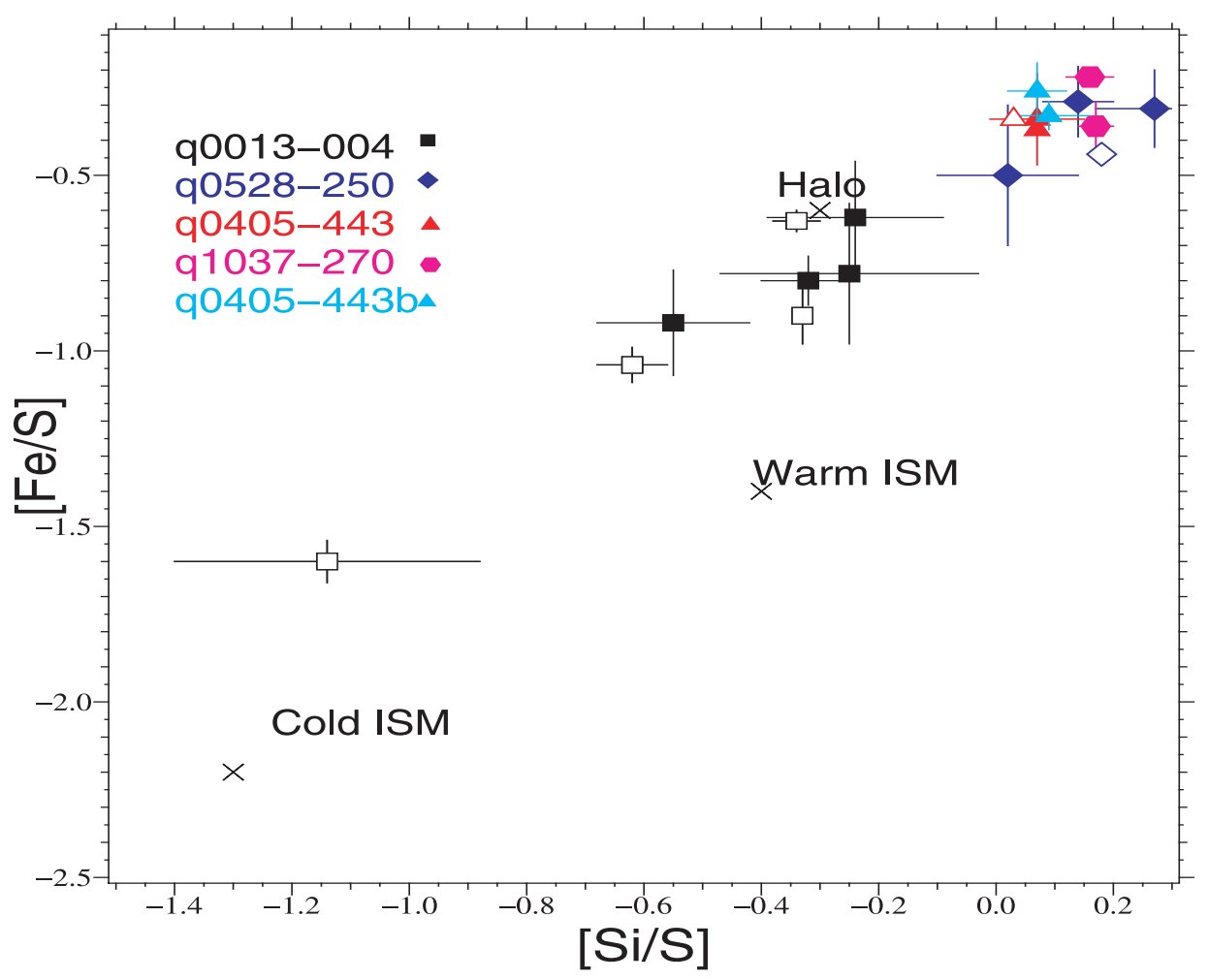

Fig. 15. $[\mathrm{Fe} / \mathrm{S}]$ vs. $[\mathrm{Si} / \mathrm{S}]$ for all the subclumps analyzed in this paper. Different symbols represent different DLA: squares for Q 0013-004, diamonds for Q 0528-250, triangles for Q 0405-443, and circles for Q 1037-270. Open symbols are used to distinguish subclumps where $\mathrm{H}_{2}$ is detected. Otherwise symbols are filled. We also indicate the typical [Fe/S] vs. [Si/S] values observed in the cold, warm ISM, and halo of our Galaxy from Welty et al. (1999).
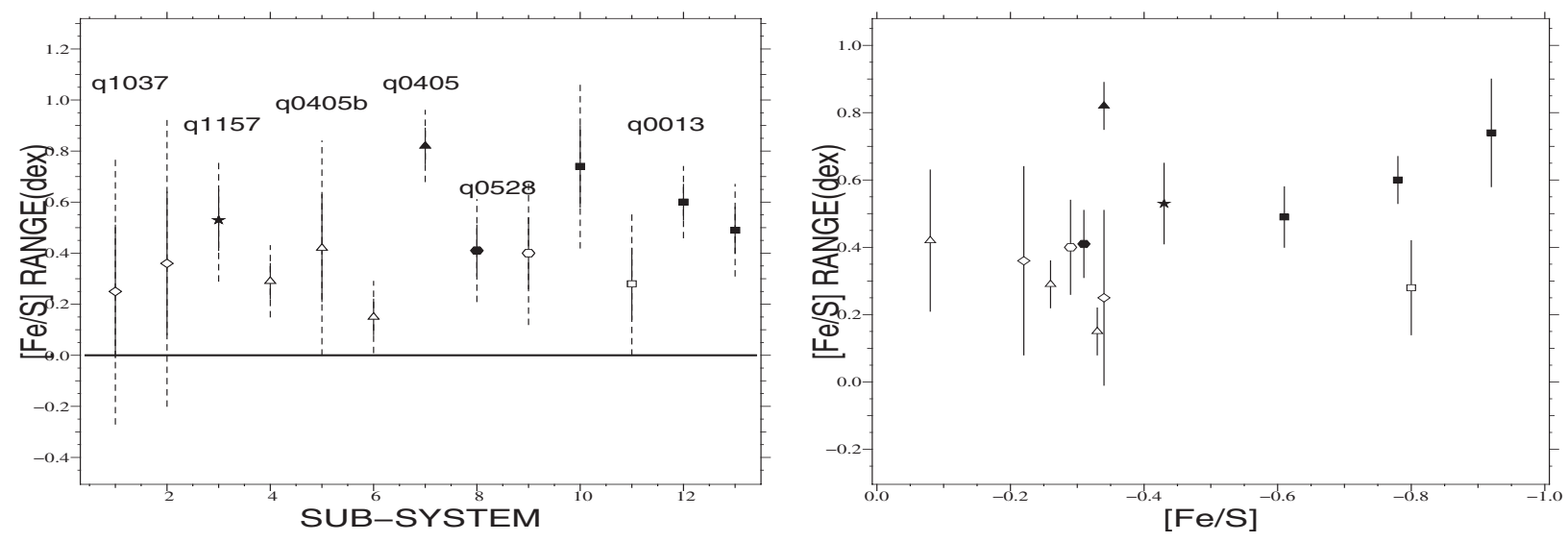

Fig. 16. Summary of the inhomogeneity amplitude observed in the subsystems. The $y$-axis represents the maximum deviation of $[\mathrm{Fe} / \mathrm{S}] \pm 2 \sigma$ in each subclump. Subsystems with molecules are respresented with filled symbols.

\subsection{Consequences}

Variations in the relative abundance pattern within DLAs are expected from different nucleosynthesis histories and from its depletion onto dust. The magnitude of variation in the nucleosynthesis pattern may depend on the history of star formation and the level of enrichment. For example, stars in the Milky Way, Magellanic clouds, and local dwarf galaxies with metallicities varying from solar to $1 / 100$ of solar show a dispersion in
[Si/Fe] of the order of 0.3 dex (e.g. Shetrone et al. 2001; Venn et al. 2001).

Peculiar nucleosynthesis histories may be reflected in the variation of abundance ratios from one subclump to the other. Although we observed differences in the relative metal abundances of different sub-clumps, they are not large. This may indicate that sub-clumps in DLAs have the same origin and history and could be part of the same object. This contrasts with the large differences in absolute metallicities that have been 
observed in LLS with similar velocity differences (e.g. D'Odorico \& Petitjean 2001).

That depletion onto dust depends on the local physical conditions should induce a large scatter in the observed pixel-topixel relative abundance ratios. The fact that only small scatter was observed may reveal that the gas in DLAs is neither very dense nor cold but rather diffuse and warm. At least the filling factor of highly depleted gas is small.

All this implies uniform physical conditions and homogeneous and efficient mixing. One can speculate that this is only possible if DLAs are small objects with dimensions on the order of one kilo-parsec. This is difficult to ascertain as direct detection of high-redshift DLAs have not been very successful till now (e.g. Kulkarni et al. 2000; Møller et al. 2004). It is, however, very important to pursue these observations in order to better constrain the nature and physical properties of these objects.

Acknowledgements. R.S. and P.P.J. gratefully acknowledge support from the Indo-French Center for the Promotion of Advanced Research (Centre Franco-Indien pour la Promotion de la Recherche Avancée) under contract No. 3004-3. This work was supported by the European Community Research and Training Network: "The Physics of the Intergalactic Medium".

\section{References}

Anders, E., \& Grevesse, N. 1989, Geochim. Cosmochim. Acta, 53, 197

Briggs, F. H., Turnshek, D. A., \& Wolfe, A. M. 1984, ApJ, 287, 549

Centuríon, M., Bonifacio, P., Molaro, P., \& Vladilo, G. 2000, ApJ, 536,540

D’Odorico, S., Cristiani, S. Dekker, H., et al. 2000, Proc. SPIE, 4005, 121

D’Odorico, V., \& Petitjean, P. 2001, A\&A, 370, 729

Ge, J., \& Bechtold, J. 1997, ApJ, 477, L73

Haehnelt, M. G., Steinmetz, M., \& Rauch, M. 1998, ApJ, 495, 647

Hou, J. L., Boissier, S., \& Prantzos, N. 2001, A\&A, 370, 23

Kanekar, N., \& Chengalur, J. N. 2003, A\&A, 399, 857
Kulkarni, V. P., Hill, J. M., Scheider, G., et al. 2000, ApJ, 536, 36

Ledoux, C., Petitjean, P., Bergeron, J., Wampler, E. J., \& Srianand, R. 1998, A\&A, 337, 51

Ledoux, C., Bergeron, J., \& Petitjean, P. 2002a, A\&A, 385, 802

Ledoux, C., Srianand, R., \& Petitjean, P. 2002b, A\&A, 392, 781

Ledoux, C., Petitjean, P., \& Srianand, R. 2003, MNRAS, 346, 209

Lespine, Y., \& Petitjean, P. 1997, A\&A, 317, 416

Lopez, S., Maza, J., Masegosa, J., \& Marquez, I. 2001, A\&A, 366, 387

Lopez, S., Reimers, D., D’Odorico, S., \& Prochaska, J. X. 2002, A\&A, 385,778

Levshakov, S. A., \& Varshalovich, D. A. 1985, MNRAS, 212, 517

Lu, L., Sargent, W. L. W., Barlow, T. A., Churchill, C. W., \& Vogt, S. S. 1996, ApJS, 107, 475

Lu, L., Savage, B. D., Tripp, T. M., \& Meyer, D. M. 1995, ApJ, 447, 597

McWilliam, A. 1997, ARA\&A, 35, 503

Møoller, P., Fynbo, J. P. U., \& Fall, M. 2004, A\&A, 422, L33

Petitjean, P., Srianand, R., \& Ledoux, C. 2002 MNRAS, 332, 383

Pettini, M., Smith, L. J., Hunstead, R. W., \& King, D. L. 1994, ApJ, 426, 79

Prochaska, J. X. 2003, ApJ, 582, 49

Prochaska, J. X., \& Wolfe, A. M. 1997, ApJ, 487, 73

Prochaska, J. X., \& Wolfe, A. M. 1999, ApJS, 121, 369

Prochaska, J. X., \& Wolfe, A. M. 2002, ApJ, 566, 68

Rachford, B. L., Snow, T. P., Tumlinson, J., et al. 2002, ApJ, 577, 221

Savage, B. D., \& Sembach, K. R. 1991, ApJ, 379, 245

Savage, B. D., \& Sembach, K. R. 1996, ARA\&A, 34, 279

Shetrone, M., Côté, P., \& Sargent, W. L. W. 2001, ApJ, 548, 592

Songaila, A., \& Cowie, L. L. 1996, AJ, 112, 335

Srianand, R., \& Petitjean, P. 1998, A\&A, 335, 33

Srianand, R., \& Petitjean, P. 2001, A\&A, 373, 816

Storrie-Lombardi, L. J., \& Wolfe, A. M. 2000, ApJ, 543, 552

Tinsley, B. M. 1979 ApJ, 229, 1046

Tumlinson, J., Shull, J. M., Rachford, B. L., et al. 2002, ApJ, 248, 460

Venn, K. A., Lennon, D. J., Kaufer, A., et al. 2001, ApJ, 547, 765

Viegas, S. M. 1995, MNRAS, 276, 268

Welty, D. E., Hobbs, L. M., Lauroesch, J. T., et al. 1999, ApJS, 124, 465

Welty, D. E., Lauroesch, J. T., Blades, J. C., Hobbs, L. M., \& York, D. G. 2001, ApJ, 554, L75

Wolfe, A. M., Turnshek, D. A., Smith, H. E., \& Cohen, R. D. 1986, ApJS, 61, 249 